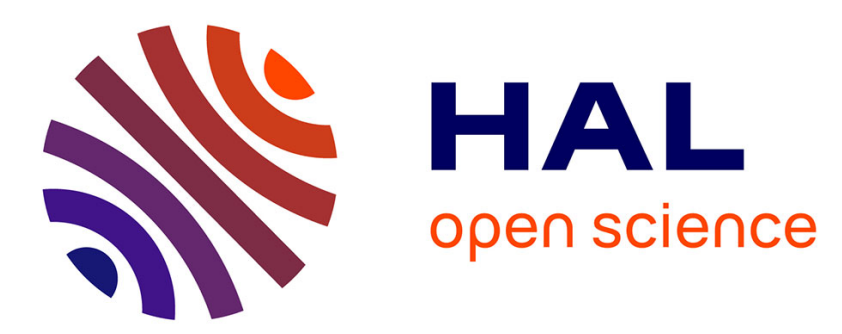

\title{
An asymptotic numerical method to solve compliant Lennard-Jones-based contact problems involving adhesive instabilities
}

Shuimiao Du, Hachmi Ben Dhia

\section{To cite this version:}

Shuimiao Du, Hachmi Ben Dhia. An asymptotic numerical method to solve compliant LennardJones-based contact problems involving adhesive instabilities. Computational Mechanics, 2019, 63, pp.1261-1281. 10.1007/s00466-018-1648-8 . hal-01898872

\section{HAL Id: hal-01898872 \\ https://hal.science/hal-01898872}

Submitted on 19 Oct 2018

HAL is a multi-disciplinary open access archive for the deposit and dissemination of scientific research documents, whether they are published or not. The documents may come from teaching and research institutions in France or abroad, or from public or private research centers.
L'archive ouverte pluridisciplinaire HAL, est destinée au dépôt et à la diffusion de documents scientifiques de niveau recherche, publiés ou non, émanant des établissements d'enseignement et de recherche français ou étrangers, des laboratoires publics ou privés. 


\title{
An asymptotic numerical method to solve compliant Lennard-Jones- based contact problems involving adhesive instabilities
}

\author{
Shuimiao Du • Hachmi Ben Dhia
}

Received: date / Accepted: date

\begin{abstract}
For compliant adhesive contact problems based on the Lennard-Jones (LJ) potential, the non-convexity of the latter leads to jump-in and jump-off instabilities which can hardly be traced by using classical algorithms. In this work, we combine an adapted Asymptotic Numerical Method (ANM) and the multiscale Arlequin method to trace efficiently these instabilities. The ANM is used to trace the entire unstable solution path in a branch-by-branch manner. The Arlequin method is used to achieve a refined resolution in the vicinity of the contact surface and to reduce possible spurious numerical oscillations due to coarse surface discretizations. Numerical results, validated by comparison with available ones, reveal the accuracy, efficiency and robustness of the proposed global methodology.
\end{abstract}

Keywords Adhesive contact · Lennard-Jones potential · Instability · Asymptotic numerical method · Multiscale · Arlequin method

\section{Introduction}

Adhesion plays an important role in many applications involving mechanical contacts at small length scales, such as that in designing and fabricating Microelectromechanical Systems (MEMS), in studying the reliability and lifetime of an Atomic Force Microscopy (AFM) probe as well as in better understanding many biological adhesive systems, to name only a few. Classical adhesion theories such as the

D. Shuimiao

Laboratoire Mécanique des Sols, Structures et Matériaux.

3 Rue Joliot Curie, 91190 Gif-sur-Yvette, France

E-mail: shuimiao.du@centralesupelec.fr

H. Ben Dhia

Laboratoire Mécanique des Sols, Structures et Matériaux.

3 Rue Joliot Curie, 91190 Gif-sur-Yvette, France

E-mail: hachmi.ben-dhia@centralesupelec.fr
Bradley theory [7], the JKR theory [22] and the DMT theory [14] provide analytical solutions for simple adhesive contact problems with idealized geometries. However, for most of the contact problems in both academic and engineering practice, the analytical solutions cannot been obtained in closed form due to various complexities. Therefore, one should resort to numerical computational methods among which the most commonly used is the Finite Element Method (FEM) in the framework of continuum mechanics combined with numerical algorithms applied for the solution of Lennard-Jones (LJ) based contact problems (see [29, $15,26,35,24,6])$. For an enlarged review of the numerical modeling methods, readers are referred to the recent survey by Sauer [28].

From a theoretical and numerical points of view, four difficulties have been pointed out in [6] regarding the resolution of the LJ-based adhesive contact problem. They are directly related to the intrinsic multi-scale, unbounded, undefined (in presence of interpenetrations) and non-convex natures of the LJ potential. The first three difficulties have been dealt with in [6] where the multiscale aspect was addressed by using the Arlequin method (see [4,5]) and the unbounded and undefined aspects were addressed by a convergent model-adaptivity method. The fourth difficulty is addressed in this work.

The nonconvexity of the LJ potential may lead to nonuniqueness of the solution, giving rise to the jump-in and jump-off adhesive instabilities (see [2,19]), especially for compliant contact problems. These adhesive instabilities have a significant influence on the measurement of the contact stiffness and surface toughness in a real experiment (see [20]), for instance. Thus they have to be accurately traced by an advanced numerical method, having in mind that this task cannot be achieved efficiently by using classical predictioncorrection methods (PCMs), such as the Newton-Raphson method. 
In this regard, the ultimate goal of the present work is to provide a computationally efficient and numerically robust method to solve LJ-based adhesive contact problems in linear elasticity. To this end, the asymptotic numerical method (ANM) proposed by Damil and Potier-Ferry [13] is adapted here to trace the jump-in and jump-off instabilities. Moreover, the Arlequin multiscale framework is employed to prevent spurious oscillations of the numerical solution due to coarse contact surface discretizations (see e.g. [27]) and to reduce computational costs. As a matter of fact, the combination of the ANM and the Arlequin method has been used to accurately capture instabilities in the buckling of rolled thin sheets under residual stresses (see [23]). In this work, the ANM-Arlequin methodology is adapted, for the first time, and tested for the solution of LJ-based unstable nonlinear and non-convex adhesive contact problems.

The paper is structured as follows. In the next section, general formulations of an obstacle-elastic frictionless contact problem based on the LJ interaction are recalled. The failure of a classical PCM in tracing instabilities of the solution of the adhesive contact problem is illustrated by a simple flat contact benchmark. In section 3, the classical ANM is adapted for the LJ-based contact problem: by introducing appropriate auxiliary fields defined on the contact surface, the strongly nonlinear and non-convex formulation of the LJ contact problem is transformed to a multifield quadratic one. An automatic continuation technique is defined to apply the ANM in a branch-by-branch manner. To enhance the global performance, a local branch dependent tolerance criterion on the relative residual norm is suggested to correct, when necessary, the accumulated equilibrium errors by distinguishing critical ending points of local branches from non critical ones. In section 4, the proposed ANM is incorporated within the Arlequin multiscale framework to improve its accuracy and efficiency. In section 5, our global solution strategy is tested for three adhesive contact problems. Concluding remarks are given in the last section.

\section{An LJ-based adhesive contact model}

\subsection{Local and weak formulations}

Consider the frictionless contact between a solid $\mathcal{B}$ occupying the closure of a bounded and regular domain $\Omega$ in $\mathbb{R}^{N_{d}}$ $\left(N_{d}=2\right.$ or 3$)$ and a rigid regular obstacle $O$ (see figure 1 ). The boundary of $\Omega$ whose unit outward normal is denoted by $\boldsymbol{n}$, is partitioned into classical parts (say, a clamped part $\Gamma_{u}$ and a part $\Gamma_{t}$ where a surface traction $t$ is prescribed) and a part $\Gamma_{c}$ where contact may occur. The interiors of these parts are assumed to be pairwise disjoint. The solid $\mathcal{B}$ is assumed to undergo linear elastic deformations. The potential contact surface of the rigid obstacle is denoted by $\Gamma_{o c}$. Under small perturbations hypotheses and neglecting friction effects, the
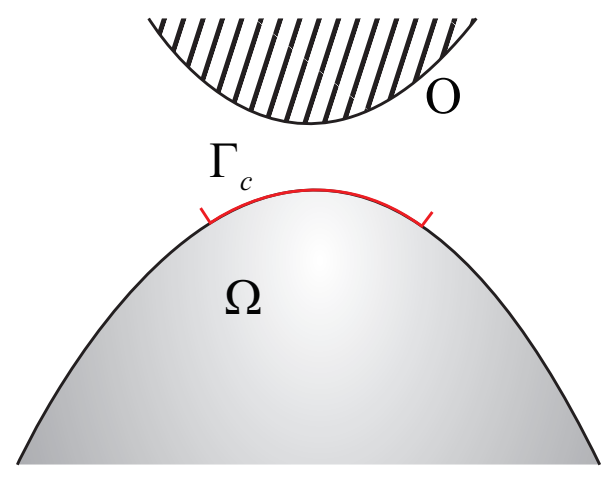

Fig. 1: An obstacle contact problem

local boundary-value problem governing the behavior of the considered mechanical system reads:

$$
\left\{\begin{array}{cc}
\operatorname{div}(\boldsymbol{\sigma})+\boldsymbol{f}_{0}=\mathbf{0} & \text { in } \Omega \\
\boldsymbol{\sigma}=\boldsymbol{R} \boldsymbol{\varepsilon} & \text { in } \Omega \\
\boldsymbol{\varepsilon}=\frac{1}{2}\left(\boldsymbol{\nabla} \boldsymbol{u}+(\boldsymbol{\nabla u})^{T}\right) & \text { in } \Omega \\
\boldsymbol{u}=\mathbf{0} & \text { on } \Gamma_{u} \\
\boldsymbol{\sigma} \boldsymbol{n}=\boldsymbol{t} & \text { on } \Gamma_{t} \\
\boldsymbol{\sigma} \boldsymbol{n}=\boldsymbol{p} \boldsymbol{n} & \text { on } \Gamma_{c}
\end{array}\right.
$$

where $\boldsymbol{\sigma}, \boldsymbol{f}_{0}, \boldsymbol{R}, \boldsymbol{\varepsilon}$ and $\boldsymbol{u}$ denote the stress tensor, the volume density of external force, the elasticity moduli, the linearised strain tensor and the displacement field, respectively.

Since the contact pressure $p$ is unknown, eq. (1) has to be supplemented with a specific contact law. In this work, we use the pressure-based LJ interaction which reads:

$p(d)=\frac{8 \Delta \gamma}{3 d_{e}}\left[\left(\frac{d_{e}}{d}\right)^{9}-\left(\frac{d_{e}}{d}\right)^{3}\right]$

where $\Delta \gamma$ denotes the relative surface energy of the contacting surfaces, $d$ denotes the signed-distance between two contacting surfaces and $d_{e}<0$ denotes the equilibrium signed-distance. The LJ interaction pressure $p(d)$ is presented in fig. 2 by the solid curve.

The signed-distance $d$ is calculated as follows. Let $\boldsymbol{x}$ denote the initial position of a material point in $\Gamma_{c}$. Then,

$$
\begin{aligned}
d & =((\boldsymbol{x}+\boldsymbol{u})-(\overline{\boldsymbol{x}}+\boldsymbol{w})) \cdot \boldsymbol{n} \\
& =(\boldsymbol{u}-\boldsymbol{w}) \cdot \boldsymbol{n}-g_{0}
\end{aligned}
$$

where $\boldsymbol{w}$ is a constant displacement prescribed to the rigid obstacle and $\overline{\boldsymbol{x}}$ refers to the paired point of $\boldsymbol{x}$ which is classically defined as the nearest point to $x$ on $\Gamma_{o c}$. The initial gap $g_{0}$ is defined by: $g_{0}=(\overline{\boldsymbol{x}}-\boldsymbol{x}) \cdot \boldsymbol{n}$.

For the approximation of this problem by means of FEM, its weak formulation is needed. It can be formally derived from eqs. (1) and (2) and reads:

Find $u \in \mathbb{V} ; \forall v \in \mathbb{V}$,

$l(\boldsymbol{v})+a(\boldsymbol{u}, \boldsymbol{v})+p_{n l}(\boldsymbol{u}, \boldsymbol{v})=0$ 


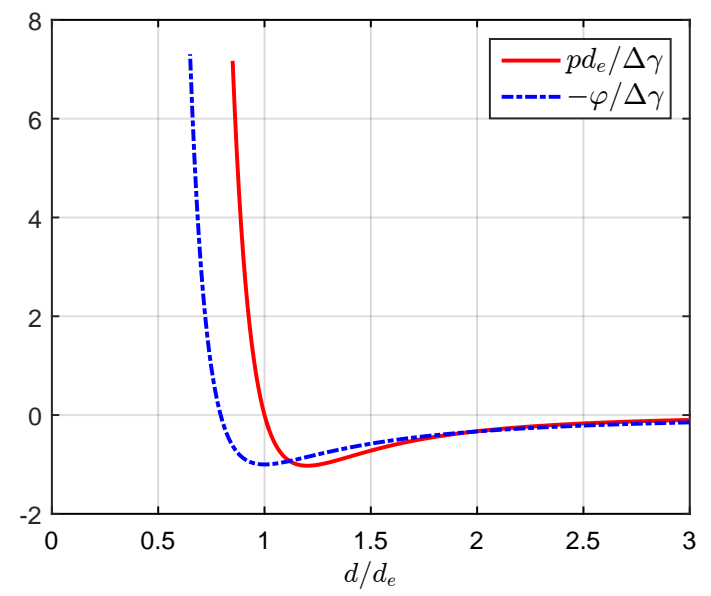

Fig. 2: The LJ potential and pressure

where $\mathbb{V}$ is the kinematically admissible field for the displacement and the quantities $l, a$ and $p_{n l}$ are linear, bilinear and nonlinear forms related to virtual works of internal, external and contact forces, respectively, defined by:

$$
\begin{aligned}
& l(\boldsymbol{v})=-\int_{\Omega} \boldsymbol{f}_{0} \cdot \boldsymbol{v}-\int_{\Gamma_{t}} \boldsymbol{t} \cdot \boldsymbol{v} \\
& a(\boldsymbol{u}, \boldsymbol{v})=\int_{\Omega} \boldsymbol{\sigma}(\boldsymbol{u}): \boldsymbol{\varepsilon}(\boldsymbol{v}) \\
& p_{n l}(\boldsymbol{u}, \boldsymbol{v})=-\int_{\Gamma_{c}} p(d(\boldsymbol{u})) \boldsymbol{n} \cdot \boldsymbol{v}
\end{aligned}
$$

Now, a question arises: how to solve the problem defined by eqs. (2), (4) and (5) efficiently? This issue is treated in what follows.

2.2 Contact instabilities and failure of the Newton-Raphson method

The LJ potential $\varphi(d)$ from which the LJ pressure eq. (2) is derived reads:

$\varphi(d)=-\frac{8 \Delta \gamma}{3}\left[\frac{1}{8}\left(\frac{d_{e}}{d}\right)^{8}-\frac{1}{2}\left(\frac{d_{e}}{d}\right)^{2}\right]$

This potential is non-convex (see the dashed curve in fig. 2), leading to possible non-uniqueness of solutions for the problem of optimization of the total potential energy from which is derived eq. (4). As a matter of fact, in a real experiment with fixed-load apparatus or in a numerical resolution with load-control technique, the non-uniqueness of solution is characterized by the onset of jump-in and jump-off instabilities occurring at the limit points on the loading and unloading branches, respectively (see e.g. [2, 19,9]). Actually, these instabilities are associated with dynamic jumps which take place in a very short time until new equilibrium is reached.
The energy loss due to the instabilities has significant influence on the measurement of contact stiffness and surface toughness (see [20]). Therefore, it is of particular interest to use a numerical method able to follow statically the entire equilibrium path and detect accurately the localization of limit points. Incidentally, this cannot be easily achieved by using classical PCMs as illustrated here by studying the following toy contact benchmark.

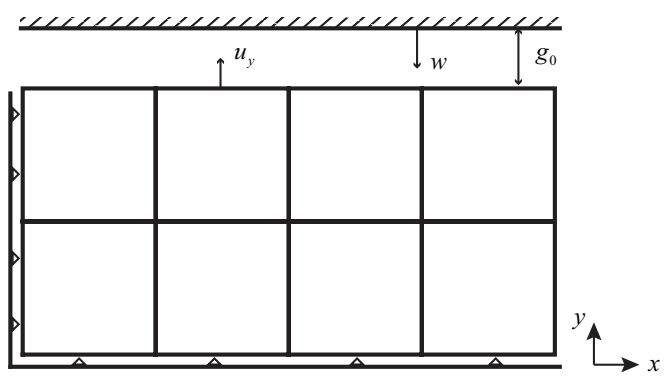

Fig. 3: A flat contact problem

Consider the adhesive contact between an elastic rectangular solid under plane strain conditions and two rigid flat planes. Taking into account the problem symmetries, only a quarter of the model is considered (see fig. 3). Let $H$ and $L$ denote the height and length of the quarter of the rectangular solid, $E$ denote the Young's modulus and let the Poisson's ratio of the material $v$ be equal to 0 . The rigid plane is separated by an initial gap $g_{0}$ from the elastic body and is given a constant displacement $w$ in the downward $y$-direction. The solution of this contact problem is governed by the following implicit function:

$f(w, d)=d+g_{0}-\frac{8 \Delta \gamma}{3 d_{e}}\left[\left(\frac{d_{e}}{d}\right)^{9}-\left(\frac{d_{e}}{d}\right)^{3}\right] \frac{H}{E}-w=0$

By using the implicit-function theorem (see e.g. [31]), one can find a critical value of the surface energy beyond which the solution develops an instability. This critical value is $\Delta \gamma_{c}=\frac{15}{72}\left(\frac{2}{15}\right)^{-\frac{2}{3}} \frac{E d_{e}^{2}}{H}$. If $\Delta \gamma<\Delta \gamma_{c}$, the adhesive interaction is too weak to produce an instability while if $\Delta \gamma>\Delta \gamma_{c}$, the adhesion is strong enough to make the solution branch unstable.

Figure 4a shows the analytical force-load curve for three values of surface energy: $\Delta \gamma=0.5 \Delta \gamma_{c}, \Delta \gamma_{c}$ and $2 \Delta \gamma_{c}$. The contact force $F$ is calculated by integrating the contact pressure along the contact surface. In this test, the geometrical and material parameters are taken as follows: $H=10 \mathrm{~nm}$, $L=20 \mathrm{~nm}, g_{0}=5 \mathrm{~nm}, E=100 G P a, d_{e}=-1 \mathrm{~nm}$. The critical surface energy is $\Delta \gamma_{c}=7.98 \mathrm{~J} / \mathrm{m}^{2}$. By a simple calculation, one can find that in the case where $\Delta \gamma=2 \Delta \gamma_{c}$, the jump-in instability occurs at $w=2.4946 \mathrm{~nm}$ while the jump-off instability occurs at $w=2.1417 \mathrm{~nm}$. 
Now let us solve this contact problem numerically. For this, it is approximated by means of FEM. Note that due to the geometrical simplicity of the domain and neglecting the Poisson's effect, the considered test can be reduced to a onedimensional problem. However, we solve it here as a twodimension problem to test the nonlinear solution method in a more general finite element framework. To this end, bilinear finite elements are used to discretize the problem (see fig. 3).

The resulting nonlinear and non-convex discrete problem is solved here by using a Damped Newton-Raphson Method where the damping technique is expected to reduce the singularity of the tangent matrix in the vicinity of the limit points. Figures $4 \mathrm{~b}$ to $4 \mathrm{~d}$ show the force-load curves for loading and unloading processes. It can be seen that when the solution is stable, the Newton's method can follow the whole loading and unloading paths. However, difficulties arise when the solution is unstable (see figs. 4c and 4d), even when very small incremental loads are used. Actually, the convergence is very slow in the zone close to the critical points. If we take the ultimate load (i.e. the one for which a convergence of the Newton algorithm can be achieved), then we find that the jump-in and jump-off instabilities occur at $2.40 \mathrm{~nm}$ and $2.21 \mathrm{~nm}$, respectively. A significant discrepancy between the obtained values and the theoretical predictions can be observed. More importantly, the $S$-shaped unstable branch is missing: the Newton's method fails to trace it.

In the following section, we present an Asymptotic Numerical Method (ANM) aimed to overcome the difficulty induced by these contact instabilities.

\section{An asymptotic numerical method to trace adhesive instabilities}

The ANM was originally developed to investigate the post-buckling of imperfect thin elastic structures (see [13]). It has a quite different philosophy with that of a classical PCM, in the sense that the latter is a pointwise scheme whilst the former is a branch-wise one. This numerical method has been used later for many other mechanical problems with moderate degree of nonlinearity. Moreover, it proves to be efficient (by comparison with more classical algorithms) in tracing statically unstable solution branches of problems with moderate nonlinearities (see e.g. [11,34,3]). Here we adapt this method to solve the LJ-based nonlinear and nonconvex adhesive contact problems.

In the following sections, we will develop all the steps for adapting the ANM to our LJ-based contact problem. In section 3.1, a "quadratic-recasting" technique is presented to transform the strongly nonlinear LJ contact problem to a quadratic one. Then, the classical ANM is applied to the resulting augmented quadratic nonlinear problem in section 3.2 and section 3.3.
3.1 Quadratic recasting of the LJ-based adhesive contact problem

For problems with a very high degree of nonlinearity, the derivation of the asymptotic formulations can be quite complex and tedious. Therefore, a process called "quadraticrecasting" (see [12]) is necessary to transform the highly nonlinear problem to a quadratic one. Here, taking advantage of the fact that the LJ pressure eq. (2) has the form of a polynomial fraction, one can reduce its nonlinearity hierarchically by introducing auxiliary fields.

Indeed, by defining the following set of supplementary fields $\boldsymbol{\theta}(\boldsymbol{x})=[s(\boldsymbol{x}), x(\boldsymbol{x}), y(\boldsymbol{x}), z(\boldsymbol{x})]$, where $\forall \boldsymbol{x} \in \Gamma_{c}$,

$\left\{\begin{array}{l}s(\boldsymbol{x}) d(\boldsymbol{x})=d_{e} \\ x(\boldsymbol{x})=s(\boldsymbol{x})^{2} \\ y(\boldsymbol{x})=x(\boldsymbol{x})^{2} \\ z(\boldsymbol{x})=y(\boldsymbol{x})^{2}\end{array}\right.$

the LJ interaction eq. (2) can be recast to a quadratic expression of the components of $\boldsymbol{\theta}$ as follows:

$p(\boldsymbol{u})=\hat{p}(\boldsymbol{\theta})=-\frac{8 \Delta \gamma}{3 d_{e}}(s z-s x)$

Then, the adhesive contact problem, governed by a strong nonlinear equation with respect to $\boldsymbol{u}$, can be transformed to a system of multifield quadratic equations with respect to $(\boldsymbol{u}, \boldsymbol{\theta})$. It reads:

$$
\left\{\begin{array}{cc}
l(\boldsymbol{v})+a(\boldsymbol{u}, \boldsymbol{v})+q(\boldsymbol{\theta}, \boldsymbol{v})=0 & \forall \boldsymbol{v} \in \mathbb{V} \\
s\left((\boldsymbol{u}-\lambda \boldsymbol{w}) \cdot \boldsymbol{n}-g_{0}\right)-d_{e}=0 & \text { on } \Gamma_{c} \\
x-s^{2}=0 & \text { on } \Gamma_{c} \\
y-x^{2}=0 & \text { on } \Gamma_{c} \\
z-y^{2}=0 & \text { on } \Gamma_{c}
\end{array}\right.
$$

where $q(\boldsymbol{\theta}, \boldsymbol{v})$ is an operator related to the virtual work of contact forces defined by:

$q(\boldsymbol{\theta}, \boldsymbol{v})=-\int_{\Gamma_{c}} \hat{p}(\boldsymbol{\theta}) \boldsymbol{n} \cdot \boldsymbol{v}$

and $\lambda$ is a loading parameter.

Now let $\overline{\boldsymbol{u}}=(\boldsymbol{u}, \boldsymbol{\theta})$ denote the new vector of unknowns containing both the original unknown $\boldsymbol{u}$ defined in the bulk and the auxiliary unknown $\boldsymbol{\theta}$ defined on the contact surface. Then the multifield quadratic problem composed of eqs. (10) and (11) can be reformulated as follows:

$\bar{c}_{0}(\boldsymbol{v})+\bar{l}_{1}(\overline{\boldsymbol{u}}, \boldsymbol{v})+\bar{q}(\overline{\boldsymbol{u}}, \boldsymbol{v})=\lambda \bar{l}_{2}(\overline{\boldsymbol{u}}, \boldsymbol{v}), \quad \forall \boldsymbol{v} \in \mathbb{V}$

with, 


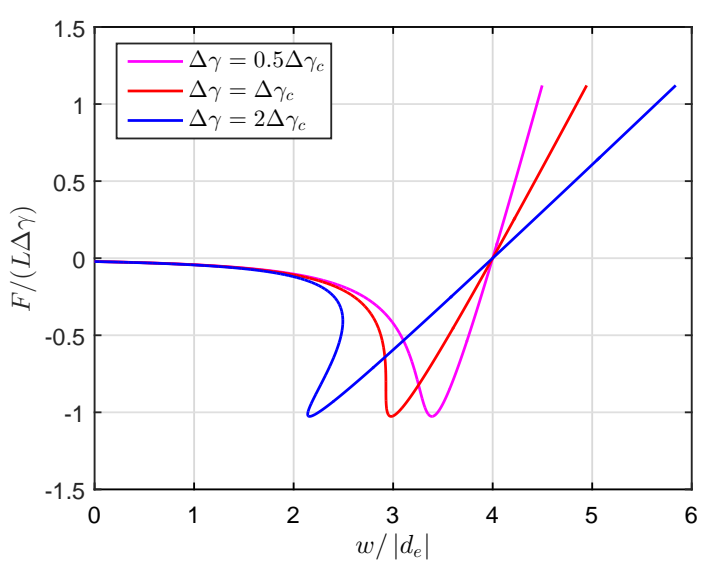

(a)

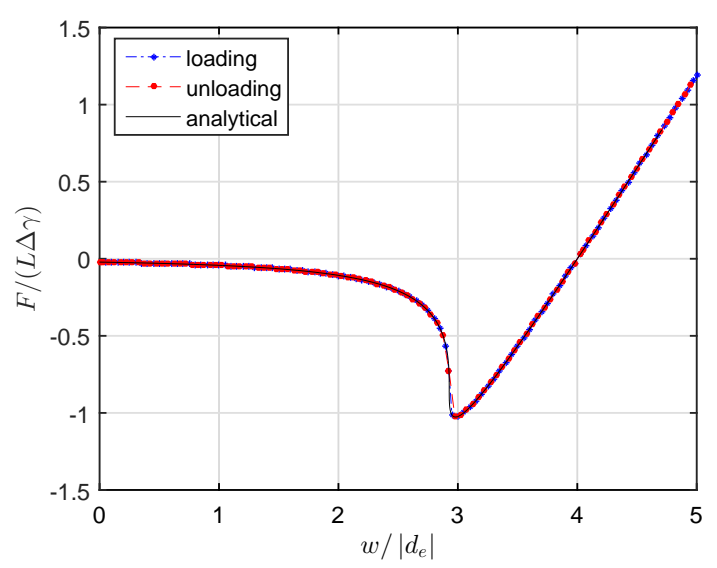

(c) $\Delta \gamma=\Delta \gamma_{c}$

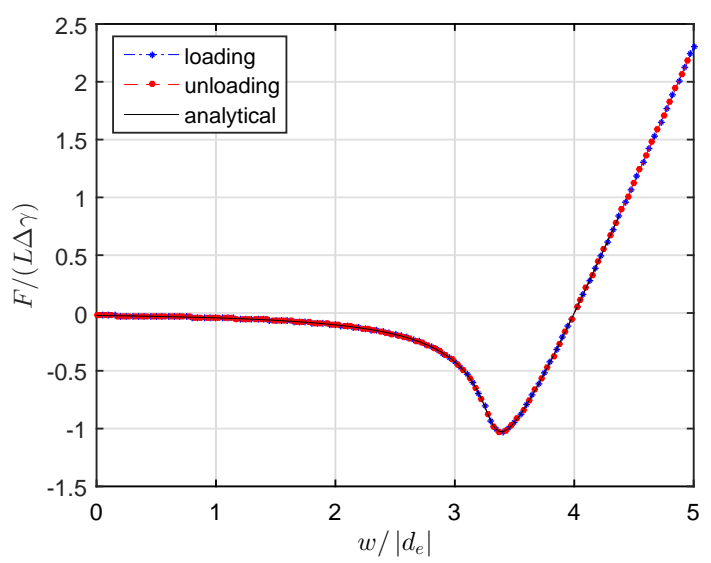

(b) $\Delta \gamma=0.5 \Delta \gamma_{c}$

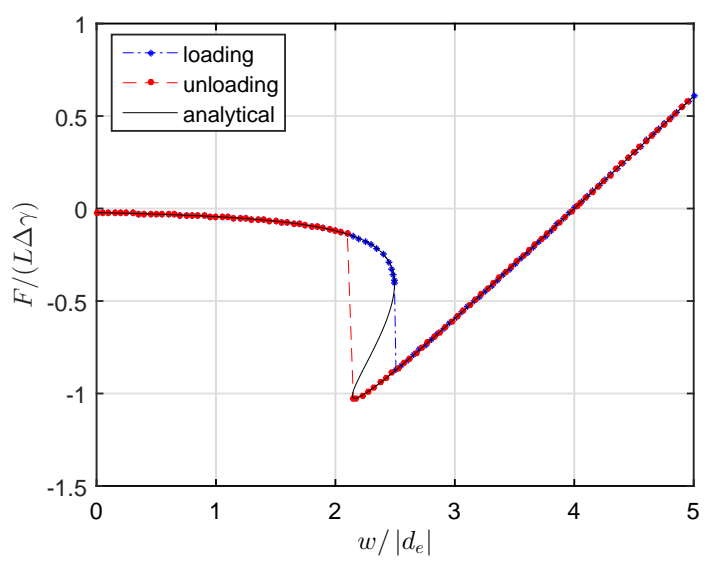

(d) $\Delta \gamma=2 \Delta \gamma_{c}$

Fig. 4: Solutions of the flat contact test with three different surface energies $\Delta \gamma$ tested, (a): analytical solution and (b)-(d): numerical solutions by using the Newton's method.

$$
\begin{array}{cc}
\bar{c}_{0}(\boldsymbol{v})=\left\{\begin{array}{c}
l(\boldsymbol{v}) \\
d_{e} \\
0 \\
0 \\
0
\end{array}\right\} & \bar{l}_{1}(\overline{\boldsymbol{u}}, \boldsymbol{v})=\left\{\begin{array}{c}
a(\boldsymbol{u}, \boldsymbol{v}) \\
s g_{0} \\
x \\
y \\
z
\end{array}\right\} \\
\bar{l}_{2}(\overline{\boldsymbol{u}}, \boldsymbol{v})=\left\{\begin{array}{c}
0 \\
-s \boldsymbol{w} \cdot \boldsymbol{n} \\
0 \\
0 \\
0
\end{array}\right\} \bar{q}(\overline{\boldsymbol{u}}, \boldsymbol{v})=\left\{\begin{array}{c}
q(\boldsymbol{\theta}, \boldsymbol{v}) \\
-s \boldsymbol{u} \cdot \boldsymbol{n} \\
-s^{2} \\
-x^{2} \\
-y^{2}
\end{array}\right\}
\end{array}
$$

The continuous problem composed of eqs. (12) and (13) is discretized by using the standard finite element method to approximate the displacement field $\boldsymbol{u}$ and a collocation method to approximate the auxiliary field $\boldsymbol{\theta}$. For this, let $\mathcal{T}_{h}$ denote a regular triangulation of the domain $\Omega$ and $\Gamma_{c h}$ denote the discrete potential contact surface. Let $\boldsymbol{U}$ denote the nodal value vector of the displacement field $\boldsymbol{u}$, which is a vector with $N_{n} \times N_{d}$ entries, where $N_{n}$ denotes the total number of nodes in $\mathcal{T}_{h}$. Each component of the auxiliary field $\boldsymbol{\theta}$ is approximated by using a collocation method. Let $\left\{p_{j}\right\}_{1 \leqslant j \leqslant N_{p}}$ be a finite collection of points in $\Gamma_{c h}$, which are tightly linked to the set of quadrature points. Let $\boldsymbol{\theta}_{j}=$ $\left[s_{j}, x_{j}, y_{j}, z_{j}\right]$ denote the value of $\boldsymbol{\theta}$ at the collocation point $p_{j}$. By using quadrature formula based on the collocation points, the surface integration eq. (11) is numerically approximated by:

$-\int_{\Gamma_{c}} \hat{p}(\boldsymbol{\theta}) \boldsymbol{n} \cdot \boldsymbol{v} \cong \frac{8 \Delta \gamma}{3 d_{e}} \sum_{j=1}^{N_{p}} w_{j}\left(s_{j} z_{j}-s_{j} x_{j}\right) \boldsymbol{n}\left(p_{j}\right) \cdot \boldsymbol{v}\left(p_{j}\right)$

where $w_{j}$ is a weight associated with the collocation point $p_{j}$.

Now, let $\boldsymbol{\Theta}=\left[\boldsymbol{\theta}_{1}, \boldsymbol{\theta}_{2}, \cdots, \boldsymbol{\theta}_{N_{p}}\right]^{T}$, a vector of $4 N_{p}$ entries, denote the global nodal value vector of $\boldsymbol{\theta}$ at all collocation points. Le us also introduce the global unknown vector 
(or the generalized coordinate vector) $\overline{\boldsymbol{U}}=[\boldsymbol{U}, \boldsymbol{\Theta}]^{T}$ whose length is $N_{n} \times N_{d}+4 N_{p}$.

Then, the discrete formulation of the adhesive contact problem reads:

$\overline{\boldsymbol{C}}_{0}+\overline{\boldsymbol{L}}_{1}(\overline{\boldsymbol{U}})+\overline{\boldsymbol{Q}}(\overline{\boldsymbol{U}}, \overline{\boldsymbol{U}})=\lambda \overline{\boldsymbol{L}}_{2}(\overline{\boldsymbol{U}})$

where $\overline{\boldsymbol{C}}_{0}, \overline{\boldsymbol{L}}_{1}, \overline{\boldsymbol{L}}_{2}$ and $\overline{\boldsymbol{Q}}$ denote the vector-valued operators related to the discretization of $\bar{c}_{0}, \bar{l}_{1}, \bar{l}_{2}$ and $\bar{q}$, respectively. In what follows, the ANM is applied to the discrete problem given by eq. (15).

\subsection{Series expansion and sequential computing}

The ANM follows the equilibrium path in polynomial branch-wise manner. So, assume that we have solved the problem eq. (15) for a given number of branches and denote by $\lambda_{0}$ the ending load of the last branch and by $\overline{\boldsymbol{U}}_{0}$ the solved displacement corresponding to $\lambda_{0}$. Observe that when necessary, a Newton-Raphson correction (see section 3.3) is applied to correct the value of $\left(\overline{\boldsymbol{U}}_{0}, \lambda_{0}\right)$ in order for the latters to satisfy (with a given tolerance) the equilibrium eq. (15). Then, we solve for the next branch by treating the possibly corrected $\left(\overline{\boldsymbol{U}}_{0}, \lambda_{0}\right)$, still denoted by $\left(\overline{\boldsymbol{U}}_{0}, \lambda_{0}\right)$, as the starting point. Now, based on a perturbation technique, the ANM assumes that the solution for the next branch $(\overline{\boldsymbol{U}}, \lambda)$ can be represented by a polynomial expansion with respect to a path parameter $a$ in the vicinity of the starting point $\left(\overline{\boldsymbol{U}}_{0}, \lambda_{0}\right)$ :

$\left\{\begin{array}{c}\overline{\boldsymbol{U}}(a)=\overline{\boldsymbol{U}}_{0}+\overline{\boldsymbol{U}}_{1} a+\overline{\boldsymbol{U}}_{2} a^{2}+\cdots+\overline{\boldsymbol{U}}_{N} a^{N} \\ \lambda(a)=\lambda_{0}+\lambda_{1} a+\lambda_{2} a^{2}+\cdots+\lambda_{N} a^{N}\end{array}\right.$

where $N$ is a truncation order.

Substituting eq. (16) into eq. (15) yields a set of algebraic equations with respect to the path parameter $a$. Each equation has a polynomial form. By equating the powers of $a$ for all orders $0 \leqslant n \leqslant N$, we obtain the following algebraic system with respect to $\left(\overline{\boldsymbol{U}}_{n}, \lambda_{n}\right)$ :

Oder $0: \overline{\boldsymbol{C}}_{0}+\overline{\boldsymbol{L}}_{1}\left(\overline{\boldsymbol{U}}_{0}\right)+\overline{\boldsymbol{Q}}\left(\overline{\boldsymbol{U}}_{0}, \overline{\boldsymbol{U}}_{0}\right)=\lambda_{0} \overline{\boldsymbol{L}}_{2}\left(\overline{\boldsymbol{U}}_{0}\right)$

Oder $1: \overline{\boldsymbol{L}}_{t}\left(\overline{\boldsymbol{U}}_{1}\right)=\lambda_{1} \overline{\boldsymbol{L}}_{2}\left(\overline{\boldsymbol{U}}_{0}\right)$

Oder $2: \overline{\boldsymbol{L}}_{t}\left(\overline{\boldsymbol{U}}_{2}\right)=\lambda_{2} \overline{\boldsymbol{L}}_{2}\left(\overline{\boldsymbol{U}}_{0}\right)+\lambda_{1} \overline{\boldsymbol{L}}_{2}\left(\bar{U}_{1}\right)-\overline{\boldsymbol{Q}}\left(\bar{U}_{1}, \bar{U}_{1}\right)$

Oder $n \geqslant 3: \overline{\boldsymbol{L}}_{t}\left(\overline{\boldsymbol{U}}_{n}\right)=\lambda_{n} \overline{\boldsymbol{L}}_{2}\left(\overline{\boldsymbol{U}}_{0}\right)$

$$
+\sum_{i=1}^{n-1} \lambda_{i} \overline{\boldsymbol{L}}_{2}\left(\overline{\boldsymbol{U}}_{n-i}\right)-\sum_{i=1}^{n-1} \overline{\boldsymbol{Q}}\left(\overline{\boldsymbol{U}}_{i}, \overline{\boldsymbol{U}}_{n-i}\right)
$$

where $\overline{\boldsymbol{L}}_{t}$ denotes the linear tangent operator at the starting point $\left(\overline{\boldsymbol{U}}_{0}, \lambda_{0}\right)$ and is defined as $\overline{\boldsymbol{L}}_{t}(\cdot)=\overline{\boldsymbol{L}}_{1}(\cdot)+2 \overline{\boldsymbol{Q}}\left(\overline{\boldsymbol{U}}_{0}, \cdot\right)-$ $\lambda_{0} \overline{\boldsymbol{L}}_{2}(\cdot)$.

Since $\left(\overline{\boldsymbol{U}}_{0}, \lambda_{0}\right)$ satisfies eq. (15) by construction, the algebraic system eq. (17) is solved sequentially (for $1 \leqslant$ $n \leqslant N)$, i.e. in an order-by-order manner. The starting point $\left(\overline{\boldsymbol{U}}_{0}, \lambda_{0}\right)$ is substituted into the first-order equation to solve for $\left(\overline{\boldsymbol{U}}_{1}, \lambda_{1}\right)$. Then, the latter is substituted into the secondorder equation to solve for $\left(\overline{\boldsymbol{U}}_{2}, \lambda_{2}\right)$. This procedure is continued until the truncation order is reached.

Incidentally, it can be seen that the linear problem at each order $1 \leqslant n \leqslant N$ is underdetermined since it consists of $N_{n} \times N_{d}+4 N_{p}+1$ unknowns but only of $N_{n} \times N_{d}+4 N_{p}$ algebraic equations. To complete the problem at each order, an additional constraint equation is introduced which relates the path parameter $a$ with the unknowns $(\bar{U}, \lambda)$. In general, the path parameter $a$ can be defined in a variety of ways. By varying the definition of $a$, traditional load-control and displacement-control techniques can be recovered as particular cases. In this work, we use the approach introduced in [11]. It consists of the projection of the increment on the tangent of the solution path and reads:

$a=\frac{1}{s_{a}^{2}}\left\langle\overline{\boldsymbol{U}}-\overline{\boldsymbol{U}}_{0}, \overline{\boldsymbol{U}}_{1}\right\rangle+\left(\lambda-\lambda_{0}\right) \lambda_{1}$

where $s_{a}$ is a strictly positive scaling parameter. Let us note that by using eq. (18), the path parameter $a$ is defined in an arc-length sense, thus both the load and displacement are controlled simultaneously (see [30,26] for the use of the arclength method to solve LJ-based contact problems with adhesive instabilities).

Substituting the asymptotic expansion eq. (16) into eq. (18) and equating the powers of $a$ yields the following additional equations:

Oder $1:\left\langle\overline{\boldsymbol{U}}_{1}, \overline{\boldsymbol{U}}_{1}\right\rangle+\lambda_{1}^{2}=s_{a}^{2}$

Oder $2 \leqslant n \leqslant N:\left\langle\overline{\boldsymbol{U}}_{n}, \overline{\boldsymbol{U}}_{1}\right\rangle+\lambda_{n} \lambda_{1}=0$

According to Equation (19), the first-order solution refers to an increment of length $s_{a}$ in the tangent direction whereas the $n$-th order solution refers to increments perpendicular to the first-order solution. The latters can be viewed as consecutive higher order corrections to the linear prediction.

Grouping eq. (17) and eq. (19), we obtain for each order $1 \leqslant n \leqslant N$ a set of linear equations and a set of quadratic (for order 1) and linear (for order $2 \leqslant n \leqslant N$ ) constraints. They read:

Oder 1: $\left\{\begin{array}{c}{\left[\boldsymbol{K}_{t}\left(\overline{\boldsymbol{U}}_{0}, \lambda_{0}\right)\right]\left\{\overline{\boldsymbol{U}}_{1}\right\}=\lambda_{1}\left\{\boldsymbol{F}_{w}\left(\overline{\boldsymbol{U}}_{0}, \lambda_{0}\right)\right\}} \\ \left\{\overline{\boldsymbol{U}}_{1}\right\}^{T}\left\{\overline{\boldsymbol{U}}_{1}\right\}+\lambda_{1}^{2}=s_{a}^{2}\end{array}\right.$

Oder $n:\left\{\begin{array}{c}{\left[\boldsymbol{K}_{t}\left(\overline{\boldsymbol{U}}_{0}, \lambda_{0}\right)\right]\left\{\overline{\boldsymbol{U}}_{n}\right\}=\lambda_{n}\left\{\boldsymbol{F}_{w}\left(\overline{\boldsymbol{U}}_{0}\right)\right\}+\left\{\boldsymbol{F}_{n l}^{n}\right\}} \\ \left\{\overline{\boldsymbol{U}}_{n}\right\}^{T}\left\{\overline{\boldsymbol{U}}_{1}\right\}+\lambda_{n} \lambda_{1}=0\end{array}\right.$ 
where $\left[\boldsymbol{K}_{t}\left(\overline{\boldsymbol{U}}_{0}, \lambda_{0}\right)\right]$ is the global tangent stiffness matrix estimated at the starting point $\overline{\boldsymbol{U}}_{0},\left\{\boldsymbol{F}_{w}\left(\overline{\boldsymbol{U}}_{0}, \lambda_{0}\right)\right\}$ is the global "force" vector related to external loads and $\left\{\boldsymbol{F}_{n l}^{n}\right\}$ is the global "force" vector referring to a measure of the accumulated nonlinear terms of preceding orders.

The global tangent matrix is calculated as follows:

$\boldsymbol{K}_{t}=\boldsymbol{K}_{E}+\boldsymbol{K}_{c}$

where $\boldsymbol{K}_{E}$ denotes the elastic stiffness matrix of the contacting body and $\boldsymbol{K}_{c}$ denotes the tangent matrix related to contact interaction defined by:

$\boldsymbol{K}_{c}=\left[\begin{array}{cc}\mathbf{0}_{N_{u} \times N_{u}} & \boldsymbol{K}_{U \Theta} \\ \boldsymbol{K}_{\Theta U} & \boldsymbol{K}_{\Theta \Theta}\end{array}\right]$

where $\boldsymbol{K}_{U \Theta}$ and $\boldsymbol{K}_{\Theta U}$ are coupling matrices linking $\boldsymbol{U}$ and $\Theta$ and $\boldsymbol{K}_{\Theta \Theta}$ is the matrix linking the auxiliary fields with each other. The matrices and vectors in eq. (20) are calculated by a classical matrix assembling technique:

$$
\begin{aligned}
& {\left[\boldsymbol{K}_{U \Theta}\right]=A_{e=1, N_{e}}\left[\boldsymbol{K}_{U \Theta}\right]^{e}} \\
& {\left[\boldsymbol{K}_{\Theta U}\right]=A_{e=1, N_{e}}\left[\boldsymbol{K}_{\Theta U}\right]^{e}} \\
& {\left[\boldsymbol{K}_{\Theta \Theta}\right]=A_{e=1, N_{e}}\left[\boldsymbol{K}_{\Theta \Theta}\right]^{e}} \\
& \left\{\boldsymbol{F}_{w}\right\}=\left\{\begin{array}{c}
\mathbf{0}_{N_{u} \times 1} \\
A_{e=1, N_{e}}\left\{\boldsymbol{F}_{w}\right\}^{e}
\end{array}\right\}, \quad\left\{\boldsymbol{F}_{n l}^{n}\right\}=\left\{\begin{array}{c}
A_{e=1, N_{e}}\left\{\boldsymbol{F}_{u}^{n}\right\}^{e} \\
A_{e=1, N_{e}}\left\{\boldsymbol{F}_{\theta}^{n}\right\}^{e}
\end{array}\right\}
\end{aligned}
$$

where $N_{e}$ denotes the number of contact elements on the contact surface, $\left[\boldsymbol{K}_{U \Theta}\right]^{e},\left[\boldsymbol{K}_{\Theta U}\right]^{e},\left[\boldsymbol{K}_{\Theta \Theta}\right]^{e}$ denote elementary tangent matrices and $\left\{\boldsymbol{F}_{u}^{n}\right\}^{e},\left\{\boldsymbol{F}_{\theta}^{n}\right\}^{e}$ denote elementary force vectors, respectively. The symbol $A$ refers to a classical matrix (or vector) assembling operator. The calculation of the above elementary matrices and vectors is detailed in appendix A for a two-node linear contact element.

Now the problem eq. (20) can be solved sequentially as follows:

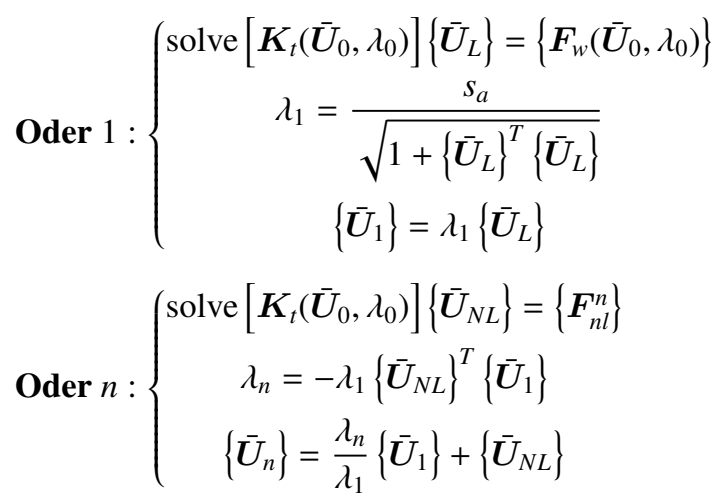

It can be seen that for each ANM step, only one global tangent matrix inversion is needed for all orders, leading to a significant gain in the computational cost compared to a classical Newton-Raphson method, for which the global tangent matrices need to be updated at each iteration.

Once the generalized coordinate vector $\bar{U}$ and the loading parameter $\lambda$ at all orders are solved by using eq. (24), the current ANM branch is re-constructed analytically with eq. (16) as expressions of the path parameter $a$.

\section{A pure displacement formulation}

It can be seen that due to auxiliary fields, the number of unknowns have increased compared with the number of Dofs for the displacement fields. Actually, by applying the same asymptotic expansion procedure to all the auxiliary fields, the relation between the contact force and the auxiliary fields at each order can be transformed back to a virtual linear interface law, leading to a purely displacementbased (still quadratic) formulation. Then, the auxiliary fields $\Theta$ are not treated directly as unknowns. They are only computed and stored intermediately for the construction of the global tangent matrix and the nonlinear RHS residual terms. The derivation of the purely displacement-based formulation is straightforward. It is not detailed here. Let us refer to Method-1 and Method-2, in the sequel, the ANM corresponding to the formulation with auxiliary fields $\Theta$ and the purely displacement-based one.

\subsection{An automatic continuation technique}

The solution of eq. (24) can only approximate the real solution path in the vicinity of the starting point. This is because the asymptotic expansion eq. (16) has a finite range of validity. This issue is illustrated by a simple test in appendix $\mathrm{B}$. Therefore, an automatic continuation technique is used here to apply the ANM in a branch-by-branch manner to trace the whole solution path. It mainly consists of three parts: the estimation of the range of validity, the estimation of the travelling direction and when necessary, the use of a Newton-Raphson correction. Moreover, an instability detection technique is also integrated herein to capture the location of the jump-in and jump-off instabilities. These points are detailed below.

\section{Estimation of the range of validity}

Two methods are commonly used to estimate the range of validity of the local asymptotic expansion eq. (16). One is based on the displacement (or generalized displacement) field. Another one is based on the residual truncation error of the equilibrium path. Throughout the present work, we 
adopt the necessary displacement criterion proposed in [11], which reads:

$\left|a_{\max }\right|=\left(\epsilon \frac{\left\|\overline{\boldsymbol{U}}_{1}\right\|}{\left\|\overline{\boldsymbol{U}}_{N}\right\|}\right)^{\frac{1}{N-1}}$

where $\epsilon$ is a tolerance number and $\|\cdot\|$ refers to the Euclidean norm.

Table 1: Estimated ranges of validity for one local branch of the solution of eq. (51). See appendix B for details.

\begin{tabular}{|c|c|c|c|c|}
\hline \multirow{2}{*}{} & \multicolumn{2}{|c|}{ Method-1 } & \multicolumn{2}{c|}{ Method-2 } \\
\cline { 2 - 5 } & $\bar{d}_{0}=4$ & $\bar{d}_{0}=1.3$ & $\bar{d}_{0}=4$ & $\bar{d}_{0}=1.3$ \\
\hline $\mathrm{N}=3$ & 0.0042 & 0.0007 & 0.0593 & 0.0002 \\
\hline $\mathrm{N}=5$ & 0.1212 & 0.0395 & 0.4058 & 0.0081 \\
\hline $\mathrm{N}=10$ & 0.7665 & 0.2778 & 1.2991 & 0.0512 \\
\hline $\mathrm{N}=15$ & 1.2875 & 0.4846 & 2.1728 & 0.0840 \\
\hline $\mathrm{N}=20$ & 1.6314 & 0.6189 & 2.0827 & 0.1052 \\
\hline
\end{tabular}

Table 1 shows the estimated ranges of validity for the problem considered in appendix $\mathrm{B}$ with a tolerance $\epsilon=10^{-7}$. The obtained results reveal a strong dependence of the range of validity on the truncation order, the starting point and whether auxiliary fields are treated as independent unknowns or not. Particularly, it can be seen that in the critical zones, Method-1 has a superior performance over Method-2 (see figs. 20b and 20d for a comparison of the quality of approximations close to the critical point).

\section{Estimation of the travelling direction}

The estimation of the range of validity eq. (25) only gives the absolute value of the path parameter but its sign remains to be determined. Indeed, the ANM solution travels towards its tangent direction (or direction of the first-order solution) if the path parameter $a$ is positive, while it travels in the inverse direction if $a$ is negative. Therefore, after passing a critical point, across which the tangent vector changes its direction, the sign of $a$ should also be inverted to avoid a trace-back to the path of the previous solution step.

Figure 5 shows the fist 15 ANM branches of the solution of eq. (51), obtained with a truncation order $N=10$ and a tolerance $\epsilon=10^{-7}$. The polynomial series are computed using Method-1 and the range of validity is estimated with criterion eq. (25). An oscillation between trace-forward and trace-back can be observed in a zone close to the critical point. This is due to a change of direction of the tangent vector (see the path from branch 9 to branch 10 and that from branch 10 to branch 11). As a consequence, the solutions are locally trapped and the critical point cannot be passed across, no matter how many branches are computed.

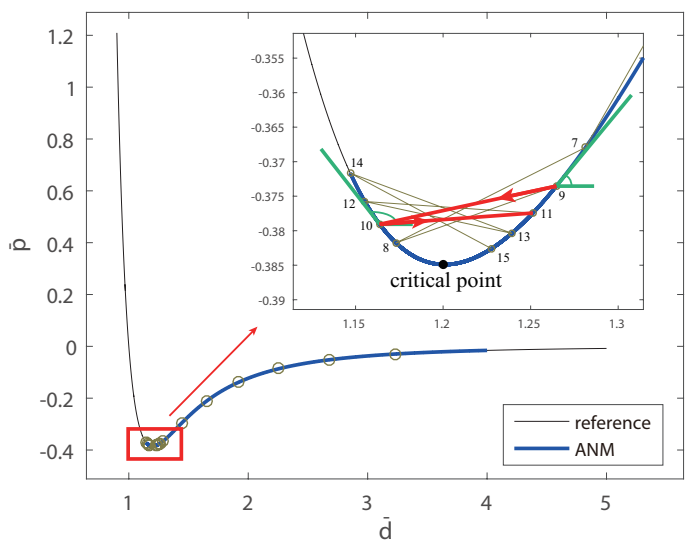

Fig. 5: Change of travelling direction in the vicinity of a critical point.

To overcome this drawback, one can reverse the travelling direction according to the sign of the product of all eigenvalues (or the determinant) of the global tangent matrix. However, in high dimensional problems, this method may be computationally inefficient. In this work, we use a method proposed in [11], which consists in evaluating the angle between the increment of the last branch and the tangent vector of the current branch. Let $\left(\overline{\boldsymbol{U}}_{+}^{j}, \lambda_{+}^{j}\right)=$ $\left(\overline{\boldsymbol{U}}\left(a_{\text {max }}^{j}\right), \lambda\left(a_{\text {max }}^{j}\right)\right)$ denote the ending point of the $j$-th solution branch. Let $\left|a_{\text {max }}^{j+1}\right|$ be the absolute value of the range of validity of the next branch, which is estimated by eq. (25). Then the travelling direction of the next branch can be evaluated as follows:

$\operatorname{sign}\left(a_{\max }^{j+1}\right)=\operatorname{sign}\left(\left\langle\overline{\boldsymbol{U}}_{+}^{j}-\overline{\boldsymbol{U}}_{+}^{j-1}, \overline{\boldsymbol{U}}_{1}^{j+1}\right\rangle+\lambda_{1}^{j+1}\left(\lambda_{+}^{j}-\lambda_{+}^{j-1}\right)\right)$

where $\left(\overline{\boldsymbol{U}}_{1}^{j+1}, \lambda_{1}^{j+1}\right)$ denotes the first-order solution of the branch $j+1$.

\section{Newton-Raphson correction}

In most cases, the estimation of the range of validity leads to a sufficiently accurate approximation of the true equilibrium branch, provided that the tolerance $\epsilon$ is chosen to be small and enough terms of the expansions are retained (see [11]). Nevertheless, it is possible that the error accumulates progressively from branch to branch and thus the approximate solution deviates more and more from the true equilibrium path. Therefore, it is necessary to estimate the global relative residual norm at the end of each ANM branch and apply a correction technique whenever it is necessary. However, as can be theoretically anticipated and has been checked numerically (see section 5), the ANM solution is only error-sensitive in the very vicinity of the critical points but not elsewhere. Taking into account this obser- 
vation, a local branch dependent tolerance criterion on the relative residual norm is introduced here for the correction technique, instead of using a global one (as is done in [32]). It reads:

$\zeta^{j}=\left|a_{\max }^{j}\right| \zeta_{g}$

where $\zeta^{j}$ denotes the tolerance of the correction at the end of branch $j$ and $\zeta_{g}$ is a global constant tolerance. In the vicinity of a critical point, $\left|a_{\text {max }}^{j}\right|$ is small (i.e. orders of magnitude smaller than 1) and the tolerance is small compared to the global one. In zones far away from a critical point, $\left|a_{\text {max }}^{j}\right|$ is relative large (i.e. of the order $O(1)$ ) and the tolerance is of the same order as the global one.

The convergence criterion for the correction phase reads:

$\boldsymbol{R}_{+}^{j} \leqslant \zeta^{j}$

where $\boldsymbol{R}_{+}^{j}$ is the relative global residual norm:

$\boldsymbol{R}_{+}^{j}=\frac{\left\|\overline{\boldsymbol{L}}_{0}+\overline{\boldsymbol{L}}_{1}\left(\overline{\boldsymbol{U}}_{+}^{j}\right)+\overline{\boldsymbol{Q}}\left(\overline{\boldsymbol{U}}_{+}^{j}, \overline{\boldsymbol{U}}_{+}^{j}\right)-\lambda_{+}^{j} \overline{\boldsymbol{L}}_{2}\left(\overline{\boldsymbol{U}}_{+}^{j}\right)\right\|}{\left\|\lambda_{+}^{j} \overline{\boldsymbol{L}}_{2}\left(\overline{\boldsymbol{U}}_{+}^{j}\right)\right\|}$

Whenever eq. (28) is not satisfied, the quantities $\left(\overline{\boldsymbol{U}}_{+}^{j}, \lambda_{+}^{j}\right)$ are corrected by using the Newton-Raphson method with an arc-length controlling technique (see [32]). The linearized system in a matrix form at each iteration $i$ reads:

$\left[\begin{array}{cc}\boldsymbol{K}_{+}^{j i} & -\boldsymbol{F}_{+}^{j i} \\ \left(\overline{\boldsymbol{U}}_{1}^{j}\right)^{T} & \lambda_{1}^{j}\end{array}\right]\left\{\begin{array}{c}\delta \overline{\boldsymbol{U}}_{+}^{j i} \\ \delta \lambda_{+}^{j i}\end{array}\right\}=\left\{\begin{array}{c}-\boldsymbol{r}_{+}^{j i} \\ 0\end{array}\right\}$

where $\boldsymbol{K}_{+}^{j i}, \boldsymbol{F}_{+}^{j i}$ and $\boldsymbol{r}_{+}^{j i}$ denote the tangent matrix, the external loading vector and the global residual vector estimated at iteration $j$, respectively. The quantities $\delta \overline{\boldsymbol{U}}_{+}^{j i}$ and $\delta \lambda_{+}^{j i}$ denotes the iterative increments of $\delta \overline{\boldsymbol{U}}_{+}^{j}$ and $\delta \lambda_{+}^{j}$ at iteration $j$, respectively. The corrected value of $\left(\overline{\boldsymbol{U}}_{+}^{j}, \lambda_{+}^{j}\right)$ is treated as a new starting point for the next branch.

The global solution strategy based on the ANM for the $\mathrm{LJ}$ adhesive contact problem is presented in algorithm 1 .

\section{Detection of contact instabilities}

The jump-in and jump-off instabilities is detected by seeking, among all ANM branches $j=1,2, \ldots$, the value of the path parameter $a_{c}^{j}$ at which the tangent of $\lambda^{j}$ vanishes, i.e. $\left.\frac{\mathrm{d} \lambda^{j}(a)}{\mathrm{d} a}\right|_{a=a_{c}^{j}}=0$, which yields the following $N$-th order polynomial equation:

$N \lambda_{N}^{j}\left(a_{c}^{j}\right)^{N-1}+(N-1) \lambda_{N-1}^{j}\left(a_{c}^{j}\right)^{N-2}+\cdots+2 \lambda_{2}^{j} a_{c}^{j}+\lambda_{1}^{j}=0$

This polynomial equation is solved by using a standard root-searching code, while rejecting the roots with an imaginary part and those lying outside the interval $\left[0, a_{\text {max }}^{j}\right]$.

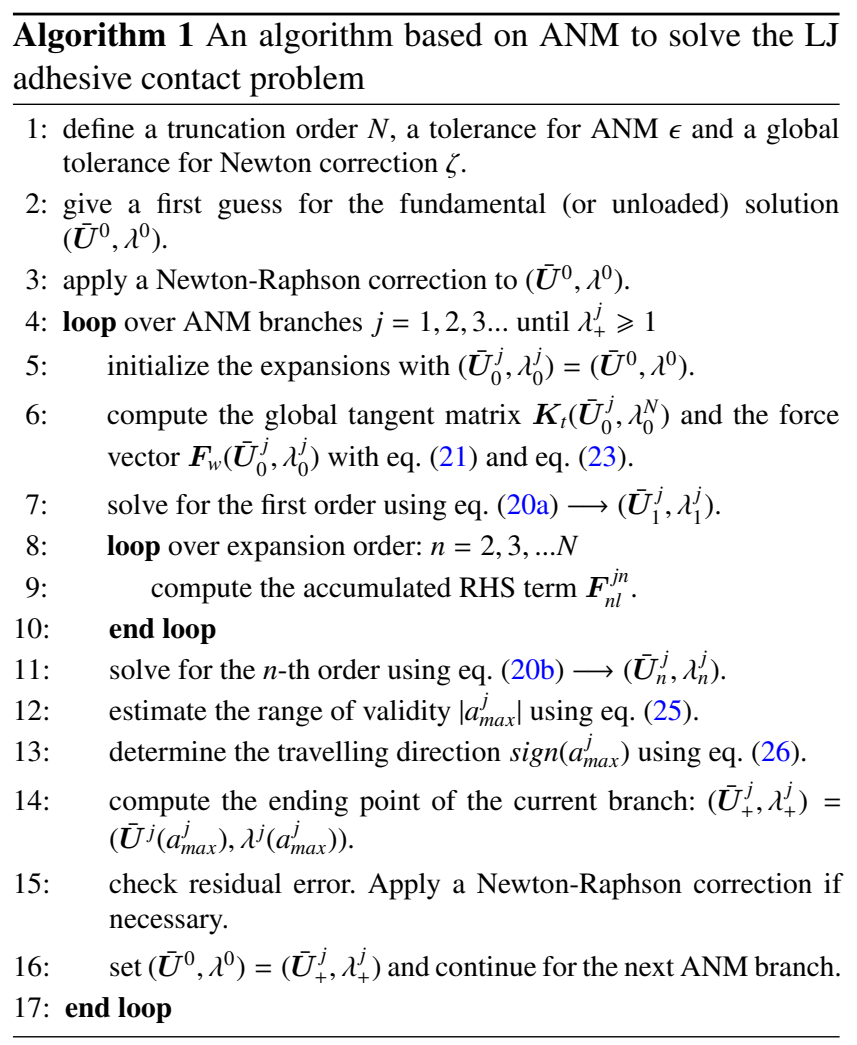

The type of instability is determined by evaluating the second-order derivative of $\lambda^{j}$ with respect to $a$ at $a_{c}^{j}$ :

$\left\{\begin{array}{l}\left.\frac{\mathrm{d}^{2} \lambda^{j}(a)}{\mathrm{d} a^{2}}\right|_{a=a_{c}^{j}}>0: \longrightarrow \text { jump-in instability } \\ \left.\frac{\mathrm{d}^{2} \lambda^{j}(a)}{\mathrm{d} a^{2}}\right|_{a=a_{c}^{j}}<0: \longrightarrow \text { jump-off instability }\end{array}\right.$

\section{Incorporation of the ANM within the Arlequin multiscale framework}

As discussed in our previous work [6], due to the very localized zones of interest and their evolution with respect to the external load, multiscale methods are highly demanded to solve the LJ-based adhesive contact problems with a reasonable computational cost. In this work, we employ again the flexible multiscale Arlequin framework (see $[4,5]$ ) to achieve this task.

Figure 6 shows an Arlequin model of the obstacleelastic contact problem presented in section 2. The domains $\Omega_{g}$ and $\Omega_{l}$ refer to the "global" and "local" domains, respectively. Their intersection is divided into $\Omega_{c}$ and $\Omega_{f}$, the former being the "coupling" zone and the latter being the "free" zone. For simplicity, we assumed that $\Gamma_{c} \subset \partial \Omega_{f}$ which is to mean that only the free-zone of the local domain is concerned by contact interactions. Then, the weak form of the LJ-based contact problem within the 

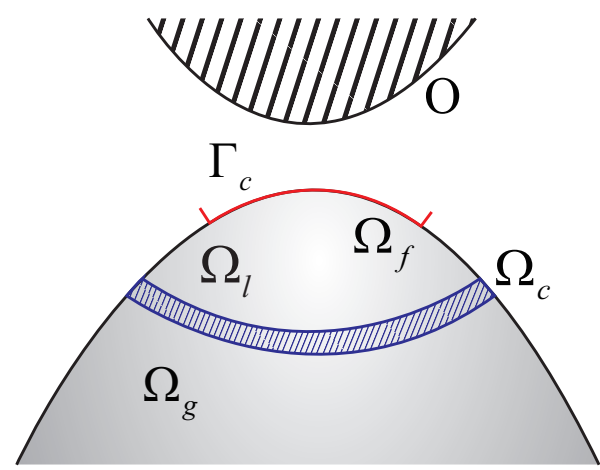

Fig. 6: Contact modeling in the Arlequin framework

Arlequin framework reads:

Find $\boldsymbol{u}_{g} \in \mathbb{V}_{g}, \boldsymbol{u}_{l} \in \mathbb{V}_{l}$, and $\phi \in \mathbb{M}$;

$\forall \boldsymbol{v}_{g} \in \mathbb{V}_{g}, \boldsymbol{v}_{l} \in \mathbb{V}_{l}$, and $\boldsymbol{\psi} \in \mathbb{M}$;

$\sum_{i=g, l} G_{i}^{i n t}\left(\boldsymbol{u}_{i}, \boldsymbol{v}_{i} ; \alpha_{i}\right)-\sum_{i=g, l} G_{i}^{e x t}\left(\boldsymbol{v}_{i} ; \beta_{i}\right)-G^{c}\left(\boldsymbol{u}_{l}, \boldsymbol{v}_{l}\right)$
$+G^{A r l}\left(\boldsymbol{u}_{g}, \boldsymbol{v}_{g}, \boldsymbol{u}_{l}, \boldsymbol{v}_{l}, \boldsymbol{\phi}, \boldsymbol{\psi}\right)=0$

where, for $i=g, l, \mathbb{V}_{i}$ denotes the kinematically admissible space for the displacement field in $\Omega_{i}$. The space $\mathrm{M}$ is the mediator space composed of the Arlequin gluing forces or volume-Lagrange multipliers $\phi$ introduced to enforce the volume coupling between the superposed models in the gluing zone $\Omega_{c}$. The quantities $\alpha_{i}$ and $\beta_{i}$ refer to Arlequin weighting functions forming a partition of unity. The quantities $G_{i}^{i n t}$ and $G_{i}^{e x t}$ refer to the weighted virtual work of internal and external forces, respectively. $G^{c}$ refers to the virtual work of contact forces and $G^{A r l}$ refers to the term related to the volume Arlequin coupling. They are calculated as follows (with prescribed surface traction being neglected): for $i=g, l$,

$G_{i}^{i n t}=\int_{\Omega_{i}} \alpha_{i} \sigma\left(\boldsymbol{u}_{i}\right): \varepsilon\left(\boldsymbol{v}_{i}\right)$

$G_{i}^{e x t}=\int_{\Omega_{i}} \beta_{i} \boldsymbol{f}_{0} \cdot \boldsymbol{v}_{i}+\int_{\Gamma_{t} \cap \partial \Omega_{i}} \beta_{i} \boldsymbol{t} \cdot \boldsymbol{v}_{i}$

$G^{c}=\int_{\Gamma_{c}} p\left(d\left(\boldsymbol{u}_{l}\right)\right) \boldsymbol{n} \cdot \boldsymbol{v}_{l}$

$G^{A r l}=C\left(\boldsymbol{u}_{g}-\boldsymbol{u}_{l}, \boldsymbol{\psi}\right)+C\left(\boldsymbol{v}_{g}-\boldsymbol{v}_{l}, \boldsymbol{\phi}\right)$

where $C$ is an Arlequin coupling operator. In this work, it is chosen to be an energy-like scalar product:

$C(\phi, u)=\int_{\Omega_{c}} \frac{E}{\kappa^{2}} \phi \cdot u+E \nabla \phi: \nabla u$

with $E$ being the material Young's modulus and $\kappa$ being a strictly positive parameter homogeneous to a length.
Following the same procedure as in the mono-model case (see section 3.1), the strongly nonlinear problem eq. (33) is transformed to a quadratic one and then discretized by the FEM and the collocation method. Let $\tilde{\boldsymbol{U}}=$ $\left[\boldsymbol{U}_{g}, \boldsymbol{U}_{l}, \boldsymbol{\Theta}_{l}, \boldsymbol{\Phi}\right]^{T}$ denote the vector of global unknowns of the problem, where $\boldsymbol{U}_{g}$ and $\boldsymbol{U}_{l}$ denote the nodal values of the global and local displacement field, $\Theta_{l}$ denote the vector of the auxiliary fields and $\boldsymbol{\Phi}$ denote the vector of the unknown Arlequin multiplier. Then, the quadratic form of the discretized problem reads:

$\tilde{C}_{0}+\tilde{\boldsymbol{L}}_{1}(\tilde{\boldsymbol{U}})+\tilde{\boldsymbol{L}}_{A r l}(\tilde{\boldsymbol{U}})+\tilde{\boldsymbol{Q}}(\tilde{\boldsymbol{U}}, \tilde{\boldsymbol{U}})=\lambda \tilde{\boldsymbol{L}}_{2}(\tilde{\boldsymbol{U}})$

where $\tilde{C}_{0}, \tilde{\boldsymbol{L}}_{1}, \tilde{\boldsymbol{L}}_{2}$ and $\tilde{\boldsymbol{Q}}$ denote constant, linear and quadratic operators similar to the ones defined in eq. (15). The operator $\tilde{\boldsymbol{L}}_{A r l}$ is a linear one related to the Arlequin coupling and defined by:

$\tilde{\boldsymbol{L}}_{A r l}(\tilde{\boldsymbol{U}})=\left[\boldsymbol{C}_{1} \boldsymbol{\Phi},-\boldsymbol{C}_{2} \boldsymbol{\Phi}, \mathbf{0}_{\operatorname{dim}(\Theta) \times 1}, \boldsymbol{C}_{1} \boldsymbol{U}_{g}-\boldsymbol{C}_{2} \boldsymbol{U}_{l}\right]^{T}$

where $C_{1}$ and $C_{2}$ denote the Arlequin coupling matrices (see [6] for details).

The resulting multiscale problem in the Arlequin framework can be solved using the ANM by following exactly the same procedure as presented in section 3 . We just give here the global tangent matrix $\boldsymbol{K}_{t}$ at each ANM branch:

$\boldsymbol{K}_{t}(\tilde{\boldsymbol{U}}, \lambda)=\left[\begin{array}{ccc}\boldsymbol{K}_{E}^{g} & \mathbf{0} & \boldsymbol{C}_{1}^{T} \\ \mathbf{0} & \boldsymbol{K}_{E}^{l}+\boldsymbol{K}_{c}^{l}-\boldsymbol{C}_{2}^{T} \\ \boldsymbol{C}_{1} & -\boldsymbol{C}_{2} & \mathbf{0}\end{array}\right]$

where $\boldsymbol{K}_{E}^{g}$ and $\boldsymbol{K}_{E}^{l}$ denote the elastic matrices in $\Omega_{g}$ and $\Omega_{l}$, respectively. The matrix $\boldsymbol{K}_{c}^{l}$ is the tangent matrix of contact interaction evaluated in the same way as that in eq. (21).

Let us note that one of the main interests of the Arlequin approach is its ability to allow for a local refinement in very localized critical zones, there where an accurate resolution is required, without alterating the global substrate model.

\section{Numerical results}

The methodology presented in this work, combining the ANM and the Arlequin method, is assessed in this section through three classical and relevant tests, for which either analytical solutions can be derived or numerical solutions are available in the literature.

\subsection{Contact between flat surfaces}

We re-consider the flat contact problem in section 2.2, for which the analytical solution has been derived (see eq. (7)) and for which it has been shown that the classical Newton-Raphson method fails to trace the jump-in and 
jump-off instabilities if the surface energy $\Delta \gamma$ exceeds the critical value $\Delta \gamma_{c}$. Here this problem is solved by using the proposed ANM with a truncation order $N=10$, an ANM tolerance $\epsilon=10^{-7}$ and a Newton-Raphson global tolerance for the correction technique $\zeta_{g}=10^{-6}$. Due to its simplicity, this toy problem is solved in a mono-model framework. Figure 7 shows the ANM solution of this con-

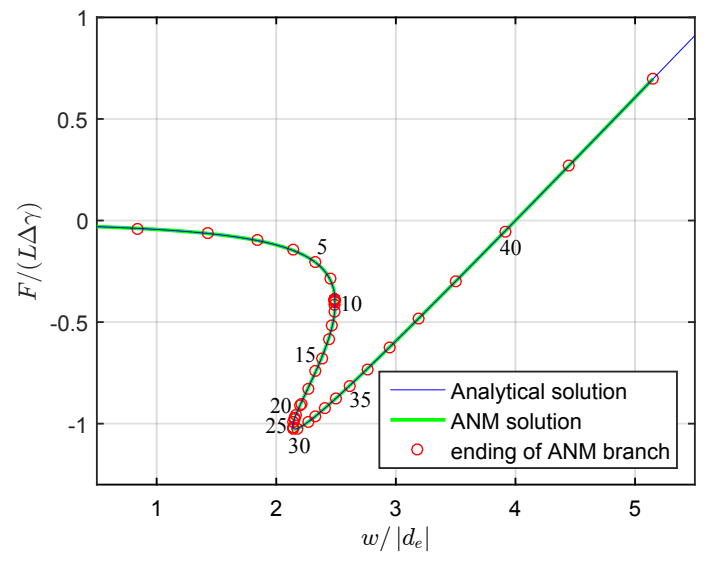

Fig. 7: ANM solution for the flat contact test with $\Delta \gamma=2 \Delta \gamma_{c}$

tact test in presence of contact instabilities $\left(\Delta \gamma=2 \Delta \gamma_{c}\right)$. The result is obtained by using Method-1. Open circles represent the ending points of ANM branches and they are labelled by the branch number for every 5 branches. The green curve is the asymptotic approximation of the real equilibrium path reconstructed by the truncated polynomial expansions eq. (16). A good agreement between the ANM solution and the analytical one is observed. Moreover, contact instabilities are also accurately traced. The ANM algorithm finds out, by using the stability detection method eqs. (31) and (32), that the jump-in instability occurs at $w=2.4946 \mathrm{~nm}$ and the jump-off instability occurs at $w=2.1417 \mathrm{~nm}$, which agrees exactly with the values predicted by analytical solutions.

In order for the external load to be fully applied, 42 ANM branches are calculated. Six Newton-Raphson iterations are also involved in the correction phase, giving rise to a total number of 48 tangent matrices inversions. We observed that these Newton-Raphson corrections are essential for the proposed algorithm to pass critical points (in the sense that no convergence of the methodology can be achieved without these corrections). A possible drift error accumulation in the vicinity of the critical point would occur otherwise. We also underline that, thanks to the new local branch dependent tolerance criterion eq. (27), only the branches in the vicinity of the critical points are concerned by the correction phase. For instance, in the considered test, the relative error at the end of branch 6 and branch 7 are
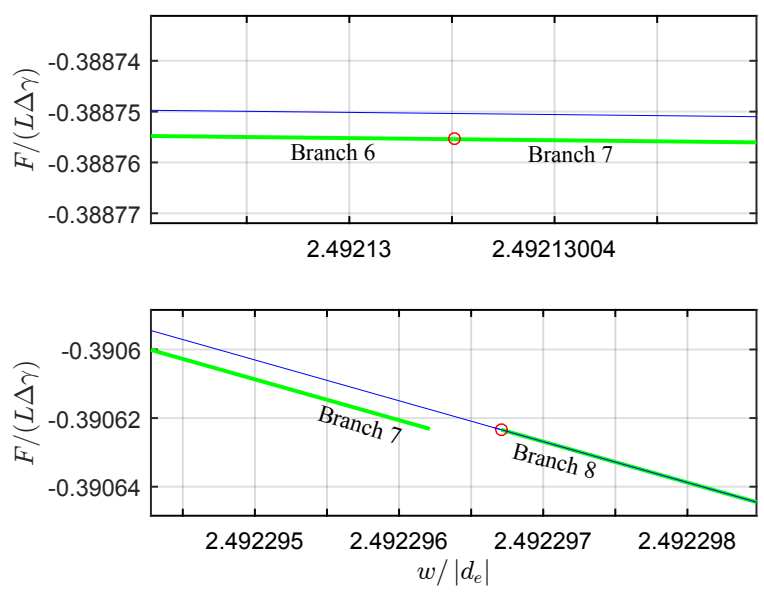

Fig. 8: Newton-Raphson correction in the vicinity of the critical point

of the same order of magnitude (about $10^{-7}$ ). However, the ending point of branch 6 is not corrected since it is not in the very vicinity of a critical point. On the contrary, the ending point of branch 7 is corrected since it is close to a jumpin critical point (see fig. 8). In this test, only about 0.14 Newton-Raphson iterations per ANM branch on average are involved as an extra computational cost: only about 1.14 matrices inversions are involved in each ANM branch, which is much more efficient than a standard Newton's method (even in the best scenarii for the latter).

Table 2: Number of matrix inversions for the flat contact test

\begin{tabular}{|c|c|c|c|}
\hline & $\Delta \gamma=0.5 \Delta \gamma_{c}$ & $\Delta \gamma=\Delta \gamma_{c}$ & $\Delta \gamma=2 \Delta \gamma_{c}$ \\
\hline Method-1 & 21 & 27 & 48 \\
\hline Method-2 & 75 & 82 & 99 \\
\hline
\end{tabular}

Table 2 shows the number of matrices inversions for the flat contact test, which is obtained as a summation of ANM branches and Newton-Raphson correction iterations. It can be seen that Method-2 is less efficient than Method-1, especially in the absence of jump-in and jump-off instabilities. This is further illustrated by fig. 9, where the estimated ranges of validity for all ANM branches are displayed for the two methods. A drop (about two orders of magnitude) of the range of validity is observed for Method-1 at the positions where a jump-in or jump-off instability occurs. In the absence of contact instabilities, no such abrupt drop of the range of validity is observed. However, for Method-2, the range of validity is considerably affected by another type of critical points, namely those with a horizontal tangent vector in the load-displacement plane. A sharp decrease (as much as about three orders of magnitude) of the range of validity is observed in the vicinity of these other kind of criti- 
cal points, no matter contact instabilities are present or not, which makes Method-2 less efficient.

As a matter of fact, the jump-in and jump-off instabilities occur only in a load-controlled test while the critical points with a horizontal tangent occur only in a displacementcontrolled test. The ANM involves a combination of both of them in an arc-length manner (with the definition of the path parameter by eq. (18)). As a consequence, any type of critical points may have more or less influence on the local performance of the ANM. These observations suggest that Method-2 is to be preferred in the vicinity of the jump-in or jump-off instabilities, while Method-1 is to be preferred in the vicinity of a critical point with a horizontal tangent. For performance considerations, one can switch between these two methods to deal with all types of critical points. However, for more complicate problems (with many local instabilities, for instance), a frequent switching between Method1 and Method-2 may lead to a loss of robustness and efficiency of the global strategy. For this reason, in the sequel, Method-1 is used for the whole path-following procedure since globally it has a better performance (see table 2).

\subsection{A spherical indentation problem: the Greenwood test}

As a second test, we consider the "Greenwood test", consisting in an adhesive contact between an infinite flat rigid surface and an elastic sphere. This test has been numerically investigated by many authors (see e.g. $[25,2,19,16])$. It is well-known that the contact in this test is governed by the Tabor parameter $\mu$ which is defined by:

$\mu=\left(\frac{R \Delta \gamma^{2}}{E^{* 2} d_{e}^{3}}\right)^{\frac{1}{3}}$

where $E^{*}$ denotes the plane-strain modulus: $E^{*}=\frac{E}{1-v^{2}}$. Indeed, for small values of $\mu$, the contact is stiff and no adhesive instabilities develop. But for large values of $\mu$, the contact is soft and jump-in and jump-off instabilities occur. In [16], the adhesive instabilities of this problem have been captured by using an arc-length continuation method in combination with analytical approaches, requiring geometrical idealizations. Here, we use the ANM and the Arlequin method to solve fully numerically this problem.

For symmetry reasons, only a quarter of the median plane of the sphere is considered. The resulting twodimensional problem is assumed to be under axisymmetric conditions. Symmetric boundary conditions are applied on the left and bottom edges of the considered domain. The radius of the disc is $R=10 \mathrm{~nm}$. The constitutive material is assumed to be elastic, isotropic and homogeneous. The Young's modulus and the Poisson's ratio are $E=100 \mathrm{GPa}$ and $v=0.3$, respectively.
Let now $\delta$ denote the signed-distance (approach) between the summit of the undeformed configuration of the sphere and the rigid plane, the initial value being $\delta_{0}=-2 n m$. A displacement $u_{0}$ in the $z$-direction is prescribed to the rigid plane surface. The equilibrium signed distance of the LJ interaction is $d_{e}=-0.25 \mathrm{~nm}$. Three tests are carried out with $\mu=0.02,0.5$ and 2, corresponding to values of the surface energy $\Delta \gamma=0.0123 \mathrm{~J} / \mathrm{m}^{2}, 1.5358 \mathrm{~J} / \mathrm{m}^{2}$ and $12.2861 \mathrm{~J} / \mathrm{m}^{2}$, respectively, the other parameters being fixed. Our numerical results obtained by the ANM are compared to the Greenwood's solutions and those obtained by using the Newton-Raphson method that we also tested here.

\section{Stable scenarii}

In a first step, the first two contact tests $(\mu=0.02$ and $\mu=0.5)$ are solved with monolithic approaches, using the mono-model mesh presented in fig. 10. The meshsize on the potential contact surface is about $h_{g}=0.3 \mathrm{~nm}$. Classical linear finite elements are used for the approximation of the displacement field and a two-node Newton-Cotes (trapezoidal) scheme is employed for the numerical integration along the contact surface (which implies that the auxiliary fields are evaluated at the vertices of the discrete contact surface).

Figures $11 \mathrm{a}$ and $11 \mathrm{~b}$ show the evolution of normalized contact force resultant $\bar{f}_{c}$ with respect to the normalized approach $\bar{\delta}$ for the Greenwood's solutions and those obtained by using the ANM and the Newton-Raphson method (denoted by "PCM" in the figure). The force resultant $\bar{f}_{c}$ and the approach $\bar{\delta}$ are normalized as follows:

$\bar{\delta}=\frac{\delta-d_{e}}{\left|d_{e}\right|}, \quad \bar{f}_{c}=\frac{f_{c}}{2 \pi \Delta \gamma R}$

For these two tests, no adhesive instabilities are detected and one can observe a good agreement between the ANM solutions and the PCM solutions. Moreover, for Tabor's parameter $\mu=0.02$, both the ANM and the PCM solutions coincide with the Greenwood's solution. But, when the contact becomes softer $(\mu=0.5)$, the discrepancy between the numerical results and the Greenwood's ones becomes larger. This can be explained by the fact that the Greenwood's paraboloidal approximation of the real circular contact surface is less accurate for large values of $\mu$.

\section{Unstable scenarii}

The scenario becomes more complicated with $\mu=2$, for which there is an onset of adhesive instabilities. Indeed, in this case, the Newton-Raphson method fails to converge by using the mesh presented in fig. 10 and we have used a finer mesh (not presented here) than the former to achieve convergence. By using the ANM, no convergence problem is encountered with the mesh presented in fig. 10 .

Figure 11c shows the force-approach curves for the Greenwood's solution, and the solution obtained by using 


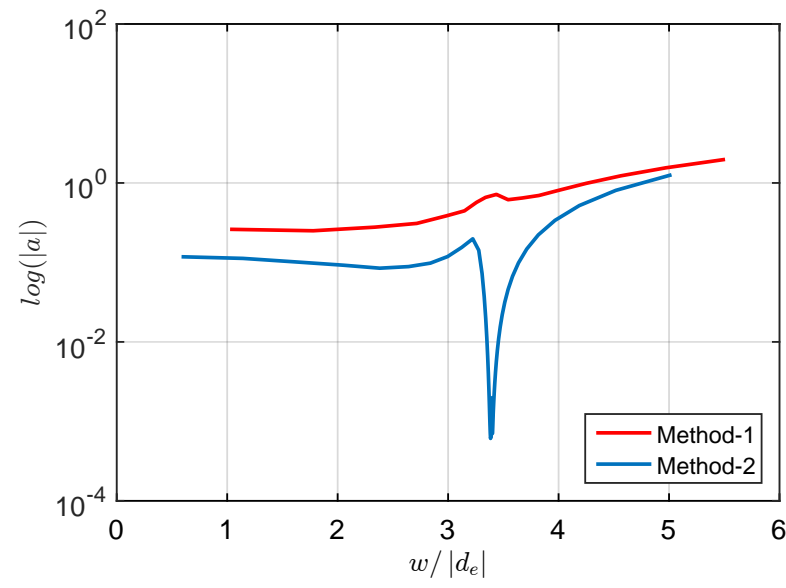

(a) $\Delta \gamma=0.5 \Delta \gamma_{c}$

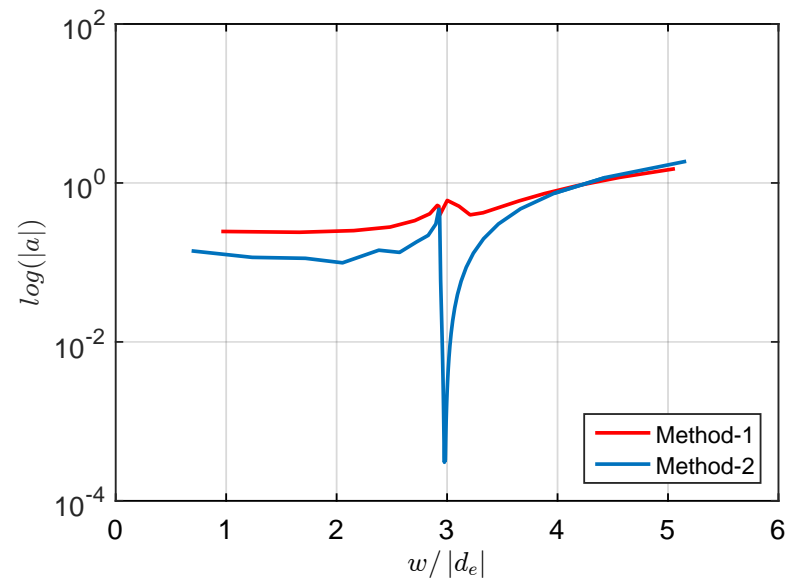

(b) $\Delta \gamma=\Delta \gamma_{c}$

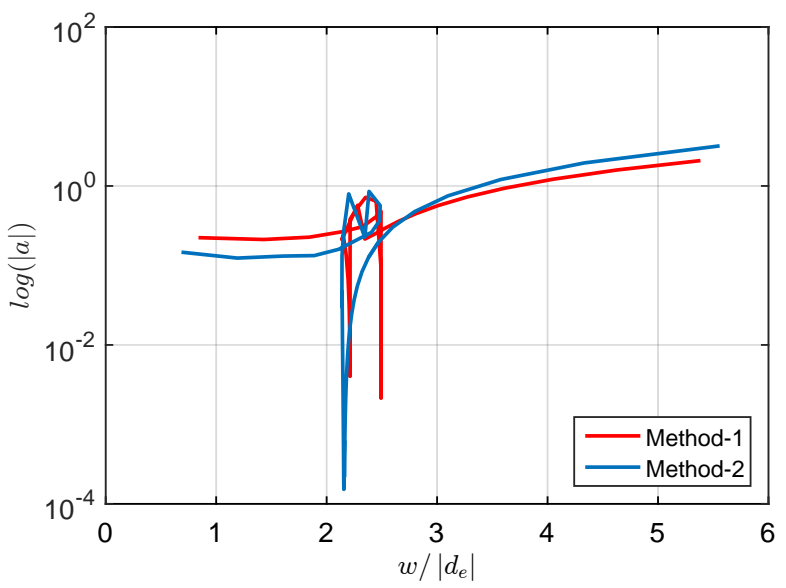

(c) $\Delta \gamma=2 \Delta \gamma_{c}$

Fig. 9: Comparison of ranges of validity for Method-1 and Method-2

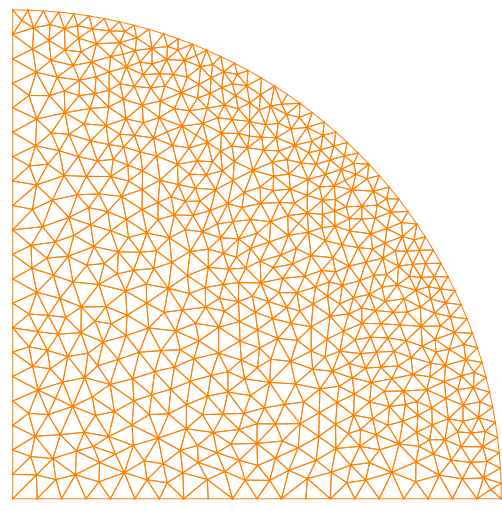

Fig. 10: Mono-model mesh of the disk

the ANM and the Newton-Raphson method (with a finer mesh). It can be seen that the physical instabilities, characterized by an $S$-shaped curve, is captured by using the ANM but not by the Newton-Raphson method. However, the ANM solution exhibits many numerical instabilities which are characterized by spurious oscillations around the mean value of the curve. These numerical instabilities are due to a too coarse contact surface discretization which does not allow for a smooth transition between adhesive and repulsive interactions. Incidentally, these numerical oscillations can explain the failure of the Newton-Raphson method in the test with the coarse mesh. They also lead to a loss of efficiency of the ANM since as can be seen in fig. 11c, an excessively large number of ANM branches are used to trace the spurious oscillations.

\section{Reduction of spurious numerical oscillations}

To reduce the aforementioned spurious numerical oscillations, we increased, in a first attempt, the number of quadrature points for the contact terms. Figure 11d shows the solution obtained by using a three-node Simpson quadrature scheme. A reduction of the amplitude of the numerical oscillations can be readily observed. However, no further re- 


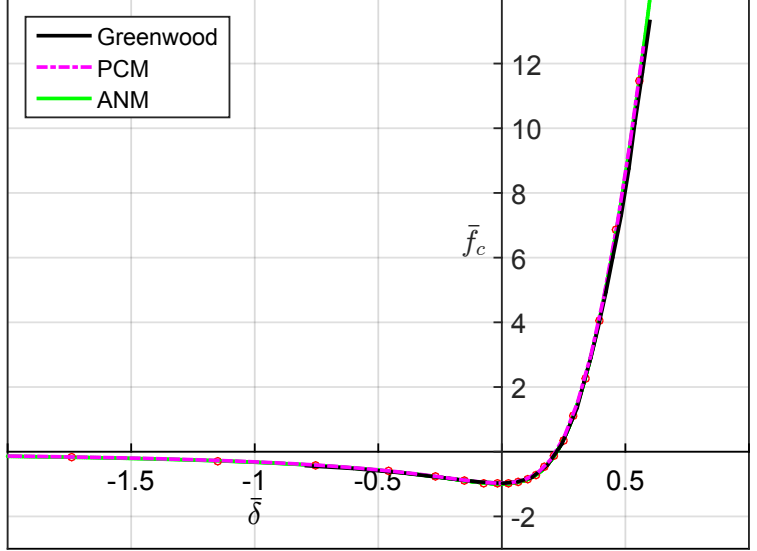

(a) $\mu=0.02$

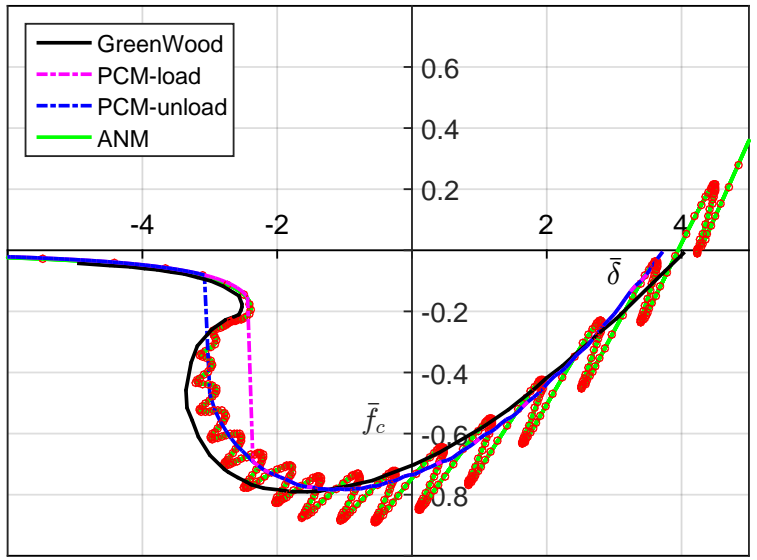

(c) $\quad \mu=2$ (Trapezoid)

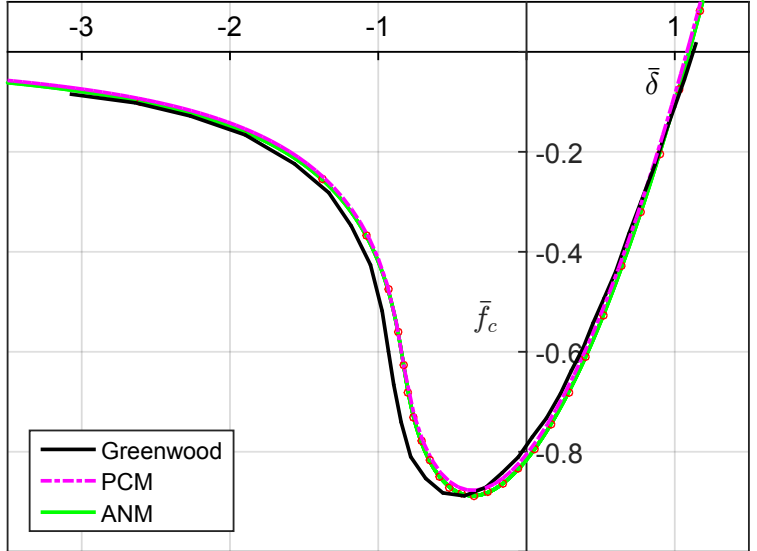

(b) $\mu=0.5$

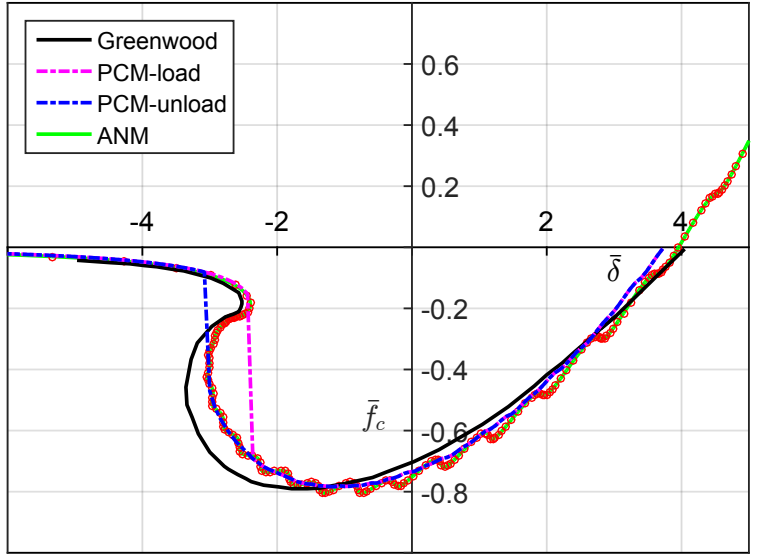

(d) $\quad \mu=2($ Simpson)

Fig. 11: Force-approach curves solved with different Tabor parameters. (a)-(b): accurate results obtained for stiff contact and (c)-(d): inaccurate results obtained for soft contact due to coarse surface discretizations

ductions were obtained by further increasing the number of quadrature points.

Considering that these numerical oscillations are due to very localized adhesive zones for the considered test, we switch to an $h$-refinement method (see e.g. [33] for an adaptive one). Bearing in mind that a monolithic $h$-refinement method may be computationally expensive, we use here the more flexible Arlequin multiscale framework to achieve this task. For this, a circular-trapezoidal patch corresponding to a central angle of $45^{\circ}$ with a thickness of $0.5 \mathrm{~nm}$ is superimposed to the global one. The meshsize $h_{l}$ of the local fine patch is about $0.03 \mathrm{~nm}$ in the vicinity of the potential contact surface. The substrate and the patch are coupled in a zone with a thickness equal to $0.25 \mathrm{~nm}$. The Arlequin weight function $\alpha_{g}$ is equal to 0.5 in $\Omega_{c}$ and to 0.01 in $\Omega_{f}$, respectively.

The remarkable improvement of the quality of the solution by using the Arlequin approach can be seen in fig. 13 .
Furthermore, the number of ANM branches and matrices inversions required to follow the whole solution path is considerably reduced: 522 and 358 matrices inversions were necessary to obtain the solutions given in fig. $11 \mathrm{c}$ and fig. 11d, respectively, whereas only 171 matrices inversions are necessary for the one given in fig. 13.

5.3 Attachment and detachment of a rigid sphere from an elastic wavy surface: the Guduru model

It is well-known that the adhesion forces decrease with respect to surface roughness (see e.g. $[18,10]$ ). However, it has been shown experimentally (see e.g. $[8,17]$ ) that in some cases, the pull-off force during the detachment of two nanoscopic contacting solids may first increase with respect to their surface roughness before eventually decreasing. Guduru $([20,21])$ suggested a theory to investigate the 


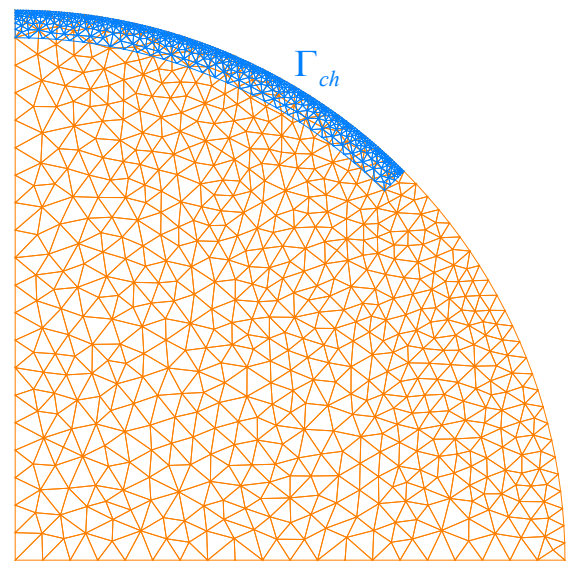

Fig. 12: Arlequin modeling: local and global meshes

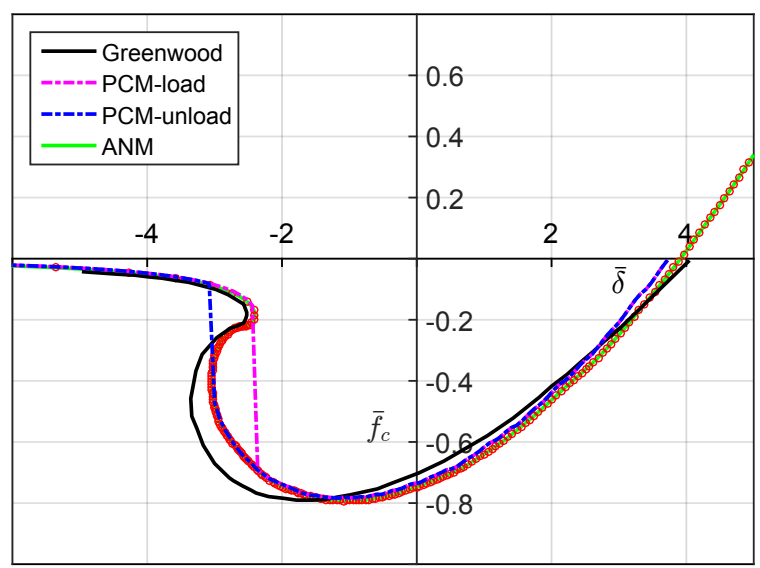

Fig. 13: Force-approach curve solved within the Arlequin framework: significant improvement can be observed

detachment of a rigid sphere from an elastic rough surface. His theory confirmed that surface roughness has significant influence on the stability behavior, which further affects the measurement of the contact stiffness and surface toughness. Here, in the last test, we assess the accuracy and efficiency of our global methodology to trace these instabilities.

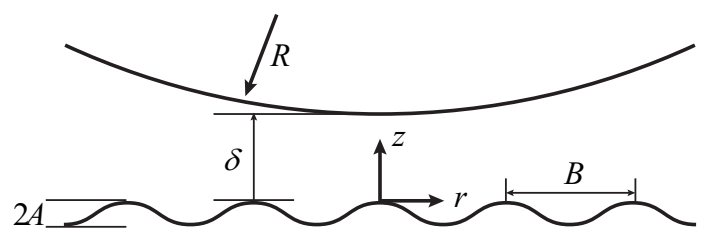

Fig. 14: Contact between a sphere and a wavy surface

In [20,21], the adhesive contact between a rigid sphere and an infinite rough surface is considered. The latter is ide- alized to be axisymmetric and in a form of single wavelength cosine function in the radial direction (see fig. 14):

$z=-A\left(1-\cos \left(\frac{2 \pi r}{B}\right)\right)$

where $r$ and $z$ are radial and vertical coordinates. The quantities $A$ and $B$ are the amplitude and the wavelength of the wavy surface. According to Guduru's theory, the approach and force are given by:

$$
\begin{aligned}
\delta\left(l_{a}\right) & =\frac{l_{a}^{2}}{R}+\frac{\pi^{2} A}{B} l_{a} H_{0}\left(\frac{2 \pi l_{a}}{B}\right)-\sqrt{\frac{2 \pi \Delta \gamma l_{a}}{E^{*}}} \\
f_{c}\left(l_{a}\right) & =\frac{2 E^{*} l_{a}^{3}}{3}\left(\frac{2}{R}+\frac{4 \pi^{2} A}{B^{2}}\right)-\sqrt{8 \pi E^{*} \Delta \gamma l_{a}^{3}} \\
& +\pi E^{*} A l_{a} H_{1}\left(\frac{2 \pi l_{a}}{B}\right)-\frac{2 E^{*} \pi^{2} A l_{a}^{2}}{B} H_{2}\left(\frac{2 \pi l_{a}}{B}\right)
\end{aligned}
$$

where $l_{a}$ denotes the macroscopic contact radius and $H_{n}(\cdot)$ denotes the Struve function of order $n$ (see [1]).

Here, two profiles of wavy surfaces are considered: $B / R=0.1, A / B=0.01$ and $B / R=0.1, A / B=0.05$. For both profiles, the other parameters are taken as follows: $R=10 \mathrm{~nm}, d_{e}=-0.25 \mathrm{~nm}$ and $\Delta \gamma=12.5 \mathrm{~J} / \mathrm{m}^{2}$. Figure 15 shows the normalized force-approach curves (parameterized by $l_{a}$ ) predicted by the Guduru's theory. The classical JKR theory for the contact between a rigid sphere and a flat contact surface is also presented. It can be seen that Guduru's prediction exhibits alternating stable and unstable local branches around the JKR prediction. Moreover, as the surface becomes rougher (in terms of amplitude), the adhesive instabilities are amplified.

Let us underline that the strong instabilities presented in fig. $15 \mathrm{~b}$ can hardly be traced accurately by using a classical PCM. Here, we solve numerically the above problem by using the proposed ANM and the Arlequin multiscale method. For this, a four-sided local patch with three straight sides and one wavy side, is superimposed to a global rectangularshaped substrate (see Figure 16 for the global and local meshes used for the simulation, in the case where $B / R=0.1$, $A / B=0.05)$. The substrate and the patch are coupled in a zone with a thickness equal to $0.25 \mathrm{~nm}$. The Arlequin weight function $\alpha_{g}$ is equal to 0.5 in $\Omega_{c}$. The paraboloidal punch, although assumed to be rigid in Guduru's theory, is also modelled and discretized here in order to assess our global solution method in a more general framework. The former is associated with an elasticity modulus several orders of magnitude larger than that of the elastic body with a wavy surface, to approximate infinite rigidity.

Figure 17 shows the obtained force-approach relation for the two tested cases. Rigorously speaking, our numerical results are not directly comparable to Guduru's results 


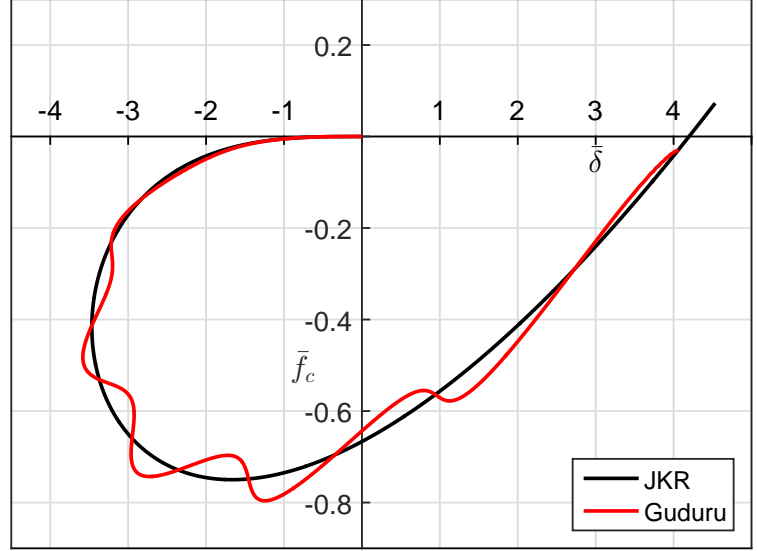

(a)

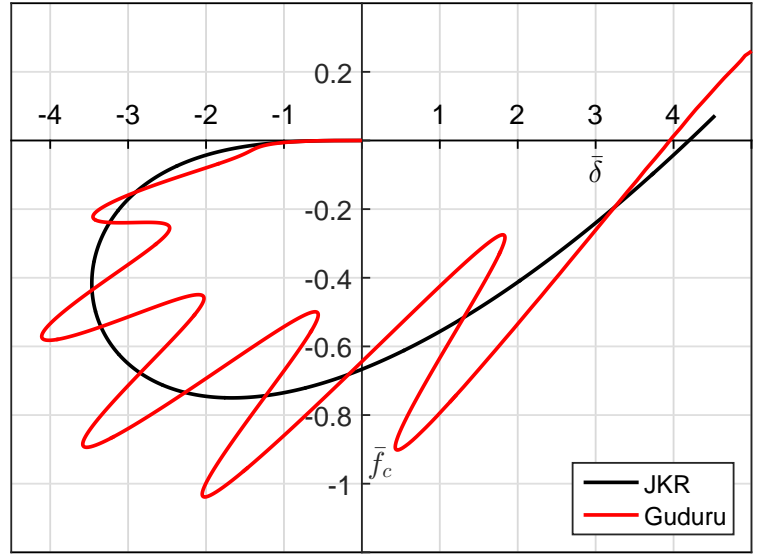

(b)

Fig. 15: Guduru's solution for the wavy surface contact: (a) $B / R=0.1, A / B=0.01$, (b) $B / R=0.1, A / B=0.05$

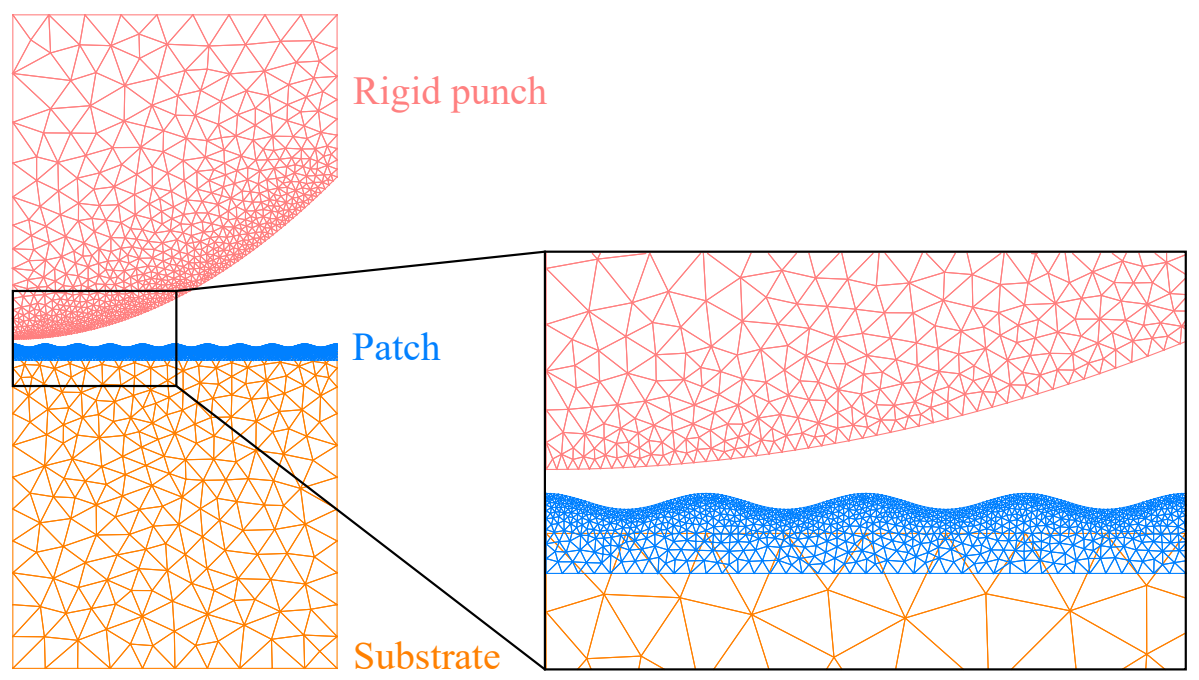

Fig. 16: Global and local finite element meshes for the wavy surface contact problem

(see appendix C) since in the latter, the atomistic interaction distance $d_{e}$ does not play any role, although this quantity does have significant influence on the adhesive contact behaviour. Nevertheless, for this test with the given parameters, a good agreement between our numerical results and Guduru's theory can still be observed during the detachment process for contact surface with moderate roughness $A / B=0.01$ (see fig. 21a and fig. 15a). As the surface roughness increases $(A / B=0.05)$, the discrepancy between our results and Guduru's theory becomes significant. This is because Guduru's theory assumes simply connected contact regions inside the contact radius, an hypothesis which is more stringent for stronger than weaker roughness. Additionally, it can be seen that our method gives more accurate results than Guduru's theory in both cases during the attachment process, where the adhesive interaction increases pro- gressively as the solids are brought together until they suddenly jump into contact (in the place where the first jump-in instability occurs). On the other hand, similar to the JKR theory, Guduru's theory is only applicable to large-scale and compliant contact problems, where the atomistic interaction distance is much smaller than the characteristic length scale of the geometry of the contacting bodies.

Observe also that, our numerical method follows the whole equilibrium path continuously, which enables one, by using a root searching procedure similar to the one used for the detection of critical points, to determine the solution not only at the ending point of each branch but also at any point on the equilibrium path. For illustration, Figure 18 shows the contact pressure and corresponding surface profiles (solid lines for deformed profiles and dashed lines for undeformed ones) at three possible equilibrium configurations labeled by 


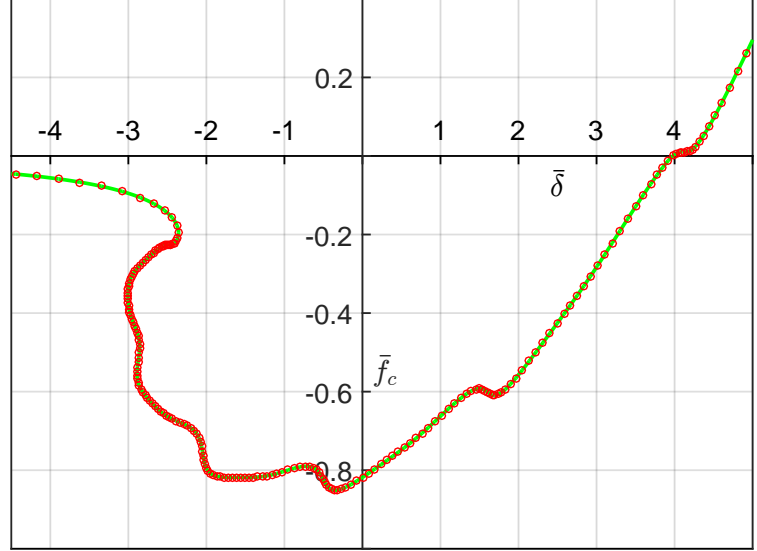

(a)

Fig. 17: ANM solution for the wavy surface contact:

$A, B$ and $C$ in fig. $21 \mathrm{~b}$ for a given approach $\bar{\delta}=1.8$. It can be readily seen that the non-uniqueness of the solution at a fixed load does come from multiple equilibrium configurations of the contact surfaces, especially at the macroscopic peeling front. These instabilities cannot be traced statically by using classical PCMs. For instance, for the classical incremental Newton-Raphson method, even when convergence is achieved, the configurations $B$ and $C$ cannot be captured in the loading process and the configurations $A$ and $B$ cannot be captured in the unloading process, leading to a failure to trace continuously the whole equilibrium path. For the proposed methodology, 197 matrices inversions are used to solve the problem with moderate roughness (see fig. 21a) and 363 matrices inversions are used to solve the one with strong roughness (see fig. 21b). A rough comparison with results reported in [6] where the Newton-Raphson method was used to solve the contact between an elastic cylinder and a rigid flat surface in absence of contact instabilities, could show the gap between the two approaches in terms of efficiency.

\section{Conclusions and perspectives}

In this paper, the jump-in and jump-off contact instabilities induced by the non-convexity of the Lennard-Jones (LJ) potential are solve here by combining appropriately an adapted Asymptotic Numerical Method (ANM) and the Arlequin multiscale framework. The resulting global solution methodology has been validated by comparing our numerical results with an analytical solution for a flat indentation test, Greenwood's solution for a spherical indentation test and Guduru's solution for a spherical detachment test with wavy surfaces. These results reveal the efficiency and robustness of the proposed methodology to trace complex

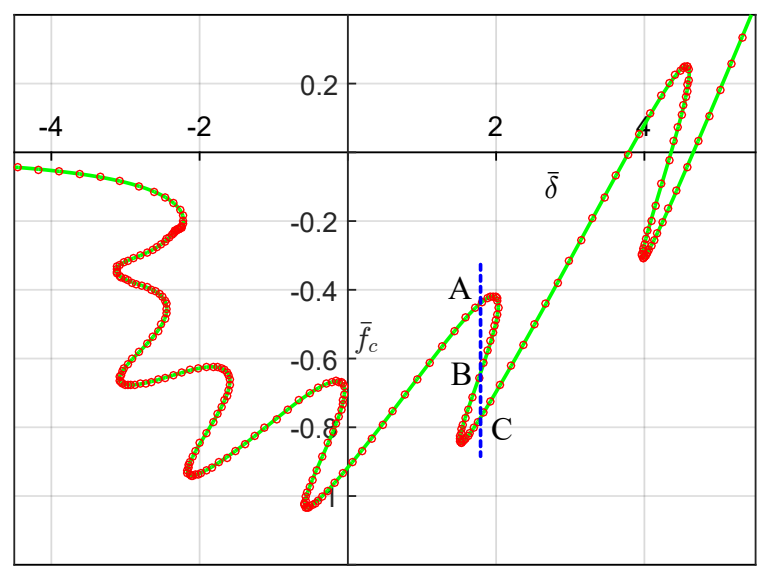

(b)

(a) $B / R=0.1, A / B=0.01$, (b) $B / R=0.1, A / B=0.05$

adhesive instabilities, at least under some appropriate hypotheses. Moreover, under the same hypotheses and as a full numerical approach, the proposed methodology can of course be applied to other LJ-based adhesive contact problems without being restricted by special forms of the contact surfaces or other ad hoc assumptions.

Concerning numerical results, only 2D adhesive contact problems have been solved in this paper. Nevertheless, by sticking to the same hypotheses used in the paper, we believe that there are no additional methodological, technical or implementation difficulties (in terms of feasibility) concerning the stability analysis of 3D LJ-based adhesive interaction problems with the adapted ANM. Finally, we restrict ourself to a couple of hypotheses such as small perturbations that may influence the instabilities picture. We believe that taking into account possible additional geometrical contact nonlinearities (leading to the change of the pairing points during the process, for instance) while keeping the ANM as methodology of analysis of the instabilities is an issue that deserves being examined farther.

\section{A Elementary matrices and vectors for a linear element}

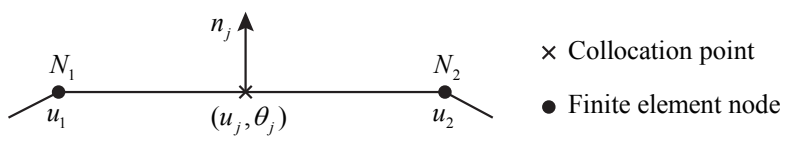

Fig. 19: A two-node linear element

Here the elementary matrices and vectors in eq. (23) are derived for a two-node linear contact element (see fig. 19). Let $N_{p e}$ denote the number of collocation points in the considered element. Let us adopt 
the following notations:

$\boldsymbol{u}_{1}=\left[u_{1}^{x}, u_{1}^{y}\right], \quad \boldsymbol{u}_{2}=\left[u_{2}^{x}, u_{2}^{y}\right]$

$\boldsymbol{n}_{j}=\left[n_{j}^{x}, n_{j}^{y}\right], \quad \boldsymbol{\theta}_{j}=\left[s_{j}, x_{j}, y_{j}, z_{j}\right], 1 \leqslant j \leqslant N_{p e}$ lows:

Let the unknowns associated with this element be arranged as fol-

$\{\overline{\boldsymbol{U}}\}^{e}=\left[\boldsymbol{u}_{1}, \boldsymbol{u}_{2}, \boldsymbol{\theta}_{1}, \cdots, \boldsymbol{\theta}_{N_{p e}}\right]^{T}$

Then the elementary matrices and vectors are calculated as follows:

$\left[\boldsymbol{K}_{U \Theta}\right]^{e}=\sum_{j=1}^{N_{p e}} w_{j}\left[\begin{array}{llll}N_{1 j} n_{j}^{x}\left(\left(z_{j}\right)_{0}-\left(x_{j}\right)_{0}\right)-N_{1 j} n_{j}^{x}\left(s_{j}\right)_{0} & 0 & N_{1 j} n_{j}^{x}\left(s_{j}\right)_{0} \\ N_{1 j} n_{j}^{y}\left(\left(z_{j}\right)_{0}-\left(x_{j}\right)_{0}\right)-N_{1 j} n_{j}^{y}\left(s_{j}\right)_{0} & 0 & N_{1 j} n_{j}^{y}\left(s_{j}\right)_{0} \\ N_{2 j} n_{j}^{x}\left(\left(z_{j}\right)_{0}-\left(x_{j}\right)_{0}\right)-N_{2 j} n_{j}^{x}\left(s_{j}\right)_{0} & 0 & N_{2 j} n_{j}^{x}\left(s_{j}\right)_{0} \\ N_{2 j} n_{j}^{y}\left(\left(z_{j}\right)_{0}-\left(x_{j}\right)_{0}\right)-N_{2 j} n_{j}^{y}\left(s_{j}\right)_{0} & 0 & N_{2 j} n_{j}^{y}\left(s_{j}\right)_{0}\end{array}\right]$

$\left[\boldsymbol{K}_{\Theta U}\right]^{e}=\sum_{j=1}^{N_{p e}} w_{j} \frac{\left(s_{j}\right)_{0}}{\left(d_{j}\right)_{0}}\left[\begin{array}{cccc}N_{1 j} n_{j}^{x} & N_{1 j} n_{j}^{y} & N_{2 j} n_{j}^{x} & N_{2 j} n_{j}^{y} \\ 0 & 0 & 0 & 0 \\ 0 & 0 & 0 & 0 \\ 0 & 0 & 0 & 0\end{array}\right]$

$\left[\boldsymbol{K}_{\Theta \Theta}\right]^{e}=\sum_{j=1}^{N_{p e}} w_{j}\left[\begin{array}{cccc}1 & 0 & 0 & 0 \\ -2\left(s_{j}\right)_{0} & 1 & 0 & 0 \\ 0 & -2\left(x_{j}\right)_{0} & 1 & 0 \\ 0 & 0 & -2\left(y_{j}\right)_{0} & 1\end{array}\right]$

$\left\{\boldsymbol{F}_{w}\right\}^{e}=\sum_{j=1}^{N_{p e}} w_{j}\left\{\begin{array}{c}\left(s_{j}\right)_{0} /\left(d_{j}\right)_{0} \boldsymbol{w} \cdot \boldsymbol{n}_{j} \\ 0 \\ 0 \\ 0\end{array}\right\}$

$\left\{\boldsymbol{F}_{u}^{n}\right\}^{e}=\sum_{j=1}^{N_{p e}} \sum_{i=1}^{n-1} w_{j}\left\{\begin{array}{l}\left(\left(z_{j}\right)_{n-i}-\left(s_{j}\right)_{i}\left(x_{j}\right)_{n-i}\right) N_{1 j} n_{j}^{x} \\ \left(\left(z_{j}\right)_{n-i}-\left(s_{j}\right)_{i}\left(x_{j}\right)_{n-i}\right) N_{1 j} n_{j}^{y} \\ \left(\left(z_{j}\right)_{n-i}-\left(s_{j}\right)_{i}\left(x_{j}\right)_{n-i}\right) N_{2 j} n_{j}^{x} \\ \left(\left(z_{j}\right)_{n-i}-\left(s_{j}\right)_{i}\left(x_{j}\right)_{n-i}\right) N_{2 j} n_{j}^{y}\end{array}\right\}$

$\left\{\boldsymbol{F}_{\theta}^{n}\right\}^{e}=\sum_{j=1}^{N_{p e}} \sum_{i=1}^{n-1} w_{j}\left\{\begin{array}{c}-\left(s_{j}\right)_{i}\left(d_{j}\right)_{n-i} /\left(d_{j}\right)_{0} \\ \left(s_{j}\right)_{i}\left(s_{j}\right)_{n-i} \\ \left(x_{j}\right)_{i}\left(x_{j}\right)_{n-i} \\ \left(y_{j}\right)_{i}\left(y_{j}\right)_{n-i}\end{array}\right\}$

where $w_{j}$ is a weight associated with the collocation point $p_{j}$, and $\left(d_{j}\right)_{i}$ is the signed-distance at point $p_{j}$ at order $i . N_{1}$ and $N_{2}$ are the standard shape functions associated with the two nodes. In all expressions, for $1 \leqslant j \leqslant N_{p e}$ and $1 \leqslant i \leqslant n$, the subscript $j$ indicates a quantity evaluated at the collocation point $p_{j}$ and the subscript $i$ outside a parenthesis indicates a quantity evaluated at order $i$.

\section{B A local ANM branch of the normalized LJ interaction}

The ANM should be applied in a branch-by-branch manner because the solution of each local branch has a finite range of validity. To illustrate this issue, let us apply the ANM to trace the equilibrium path of the following single algebraic equation:

$\bar{p}=\left(\frac{1}{\bar{d}}\right)^{9}-\left(\frac{1}{\bar{d}}\right)^{3}$

Equation (51) is a normalized form of the $\mathrm{LJ}$ interaction eq. (2), where the signed-distance and the contact pressure are normalized as $\bar{d}=\frac{d}{d_{e}}$ and $\bar{p}=p \frac{3 d_{e}}{8 \Delta \gamma}$, respectively. Two different initializations corresponding to $\bar{d}_{0}=4$ and $\bar{d}_{0}=1.3$ are tested and different truncation orders $N=$ $3,5,10,15$ and 20 are tested. Numerical experiments are conducted separately using Method-1 and Method-2 depending whether auxiliary fields are treated as independent unknowns or not.

Figure 20 shows the solution of one ANM branch, where in figs. 20a and 20c, each curve is plotted for $a$ varies from 0 to 3 and in fig. 20b, $a$ varies from 0 to 1.25 for all curves. However, in fig. 20d, the values of $a$ at the ending point for each curve is not the same (which varies from 0.2 to 0.3 ) due to a too rapid deviation from the reference curve. It can be seen that in all cases, the solution has a finite range of validity since the approximation is only acceptable up to a finite value of $a$. Beyond a certain step length $a$, the solution deviates rapidly from the real solution path. All the factors considered (i.e. the truncation order, starting point, whether Method-1 or Method-2 is used, etc.) have significant influence on the range of validity. Indeed, the starting point indicates the local nonlinearity of the equilibrium path. At the starting point $\bar{d}_{0}=4$, the local nonlinearity is moderate since the contribution of the term $\left(\frac{1}{d}\right)^{9}$ can be neglected, thus a large step size is adequate for an accurate approximation. Moreover, in this case with a moderate local nonlinearity, using Method-1 or Method- 2 makes no large difference on the solution. However, at the starting point $\bar{d}_{0}=1.3$, a very strong local nonlinearity is encountered and the range of validity is drastically reduced. In such a case with a strong local nonlinearity, Method-1 is preferred to give a relatively large range of validity. In all cases considered, augmenting the truncation order can increase the range of validity. However, beyond order 15 , continuing augmenting the truncation order does not make much improvement of the solution since very high-order corrections are too small in magnitude.

\section{Comparison of the ANM results with the Guduru theory}

Figure 21 shows the comparison of our numerical results, obtained with the asymptotic numerical and the Arlequin methods, with the results predicted within the Guduru theory for the weakly and the strongly wavy surfaces, considered in section 5.3. One can see the significant difference between the results obtained by the two approaches, especially for strongly wavy surface. This stems clearly from the fact that the model assumptions on which rely the two approaches are different. Actually, Guduru's theory assumes, in particular, a continuous contact zone. The latter hypothesis is not accurate, especially for the strongly wavy surface. Moreover, since the Guduru theory is derived from the JKR one, it does not include adhesive interaction for large separations, as can be seen in fig. 21 .

\section{References}

1. Abramowitz, M., Stegun, I.A.: Handbook of mathematical functions with formulas, graphs, and mathematical table, vol. 2172. Dover New York (1965) 
2. Attard, P., Parker, J.L.: Deformation and adhesion of elastic bodies in contact. Physical Review A 46(12), 7959 (1992)

3. Baguet, S., Cochelin, B.: On the behaviour of the anm continuation in the presence of bifurcations. International Journal for Numerical Methods in Biomedical Engineering 19(6), 459-471 (2003)

4. Ben Dhia, H.: Multiscale mechanical problems: the arlequin method. Comptes Rendus de l'Academie des Sciences Series IIB Mechanics Physics Astronomy 326(12), 899-904 (1998)

5. Ben Dhia, H.: Numerical modelling of multiscale problems: the arlequin method. CD Proceedings of ECCM'99, Munchen (1999)

6. Ben Dhia, H., Du, S.: A model-adaptivity method for the solution of lennard-jones based adhesive contact problems. Computational Mechanics pp. 1-20 (2018)

7. Bradley, R.S.: The cohesive force between solid surfaces and the surface energy of solids. The London, Edinburgh, and Dublin Philosophical Magazine and Journal of Science 13(86), 853-862 (1932)

8. Briggs, G., Briscoe, B.: The effect of surface topography on the adhesion of elastic solids. Journal of Physics D: Applied Physics 10(18), 2453 (1977)

9. Cappella, B., Dietler, G.: Force-distance curves by atomic force microscopy. Surface science reports 34(1-3), 15-3104 (1999)

10. Chiche, A., Pareige, P., Creton, C.: Role of surface roughness in controlling the adhesion of a soft adhesive on a hard surface. Comptes Rendus de l'Académie des Sciences-Series IV-Physics 1(9), 1197-1204 (2000)

11. Cochelin, B.: A path-following technique via an asymptoticnumerical method. Computers \& structures 53(5), 1181-1192 (1994)

12. Cochelin, B., Vergez, C.: A high order purely frequency-based harmonic balance formulation for continuation of periodic solutions. Journal of sound and vibration 324(1-2), 243-262 (2009)

13. Damil, N., Potier-Ferry, M.: A new method to compute perturbed bifurcations: application to the buckling of imperfect elastic structures. International Journal of Engineering Science 28(9), 943957 (1990)

14. Derjaguin, B.V., Muller, V.M., Toporov, Y.P.: Effect of contact deformations on the adhesion of particles. Journal of Colloid and interface science 53(2), 314-326 (1975)

15. Du, Y., Chen, L., McGruer, N.E., Adams, G.G., Etsion, I.: A finite element model of loading and unloading of an asperity contact with adhesion and plasticity. Journal of colloid and interface science 312(2), 522-528 (2007)

16. Feng, J.Q.: Contact behavior of spherical elastic particles: a computational study of particle adhesion and deformations. Colloids and Surfaces A: Physicochemical and Engineering Aspects 172(1), 175-198 (2000)

17. Fuller, K., Roberts, A.: Rubber rolling on rough surfaces. Journal of Physics D: Applied Physics 14(2), 221 (1981)

18. Fuller, K., Tabor, D.: The effect of surface roughness on the adhesion of elastic solids. Proc. R. Soc. Lond. A 345(1642), 327-342 (1975)

19. Greenwood, J.: Adhesion of elastic spheres. In: Proceedings of the Royal Society of London A: Mathematical, Physical and Engi- neering Sciences, vol. 453(1961), pp. 1277-1297. The Royal Society (1997)

20. Guduru, P.: Detachment of a rigid solid from an elastic wavy surface: theory. Journal of the Mechanics and Physics of Solids 55(3), 445-472 (2007)

21. Guduru, P., Bull, C.: Detachment of a rigid solid from an elastic wavy surface: experiments. Journal of the Mechanics and Physics of Solids 55(3), 473-488 (2007)

22. Johnson, K., Kendall, K., Roberts, A.: Surface energy and the contact of elastic solids. In: Proceedings of the Royal Society of London A: Mathematical, Physical and Engineering Sciences, vol. 324(1558), pp. 301-313. The Royal Society (1971)

23. Kpogan, K., Zahrouni, H., Potier-Ferry, M., Dhia, H.B.: Buckling of rolled thin sheets under residual stresses by anm and arlequin method. International Journal of Material Forming 10(3), 389-404 (2017)

24. Medina, S., Dini, D.: A numerical model for the deterministic analysis of adhesive rough contacts down to the nano-scale. International Journal of Solids and Structures 51(14), 2620-2632 (2014)

25. Muller, V., Yushchenko, V., Derjaguin, B.: On the influence of molecular forces on the deformation of an elastic sphere and its sticking to a rigid plane. Journal of Colloid and Interface Science 77(1), 91-101 (1980)

26. Radhakrishnan, H., Mesarovic, S.D.: Adhesive contact of elastic spheres revisited: numerical models and scaling. In: Proceedings of the Royal Society of London A: Mathematical, Physical and Engineering Sciences, vol. 465, pp. 2231-2249. The Royal Society (2009)

27. Sauer, R.A.: Enriched contact finite elements for stable peeling computations. International Journal for Numerical Methods in Engineering 87(6), 593-616 (2011)

28. Sauer, R.A.: A survey of computational models for adhesion. The Journal of Adhesion 92(2), 81-120 (2016)

29. Sauer, R.A., Li, S.: An atomic interaction-based continuum model for computational multiscale contact mechanics. PAMM 7(1), 4080,029-4080,030 (2007)

30. Sauer, R.A., Li, S.: A contact mechanics model for quasi-continua. International journal for numerical methods in engineering 71(8), 931-962 (2007)

31. Taylor, A.E., Mann, W.R.: Advanced calculus. Wiley (1972)

32. Vannucci, P., Cochelin, B., Damil, N., Potier-Ferry, M.: An asymptotic-numerical method to compute bifurcating branches. International journal for numerical methods in engineering 41(8), 1365-1389 (1998)

33. Wriggers, P.: Finite element algorithms for contact problems. Archives of Computational Methods in Engineering 2(4), 1-49 (1995)

34. Zahrouni, H., Cochelin, B., Potier-Ferry, M.: Computing finite rotations of shells by an asymptotic-numerical method. Computer methods in applied mechanics and engineering 175(1-2), 71-85 (1999)

35. Zhang, X., Zhang, X., Wen, S.: Finite element modeling of the nano-scale adhesive contact and the geometry-based pull-off force. Tribology letters 41(1), 65-72 (2011) 


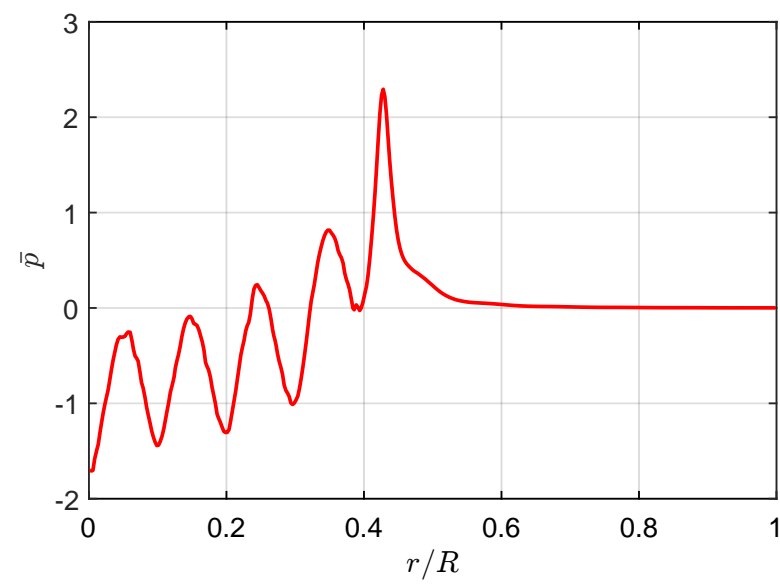

(a) contact force: config. $A$

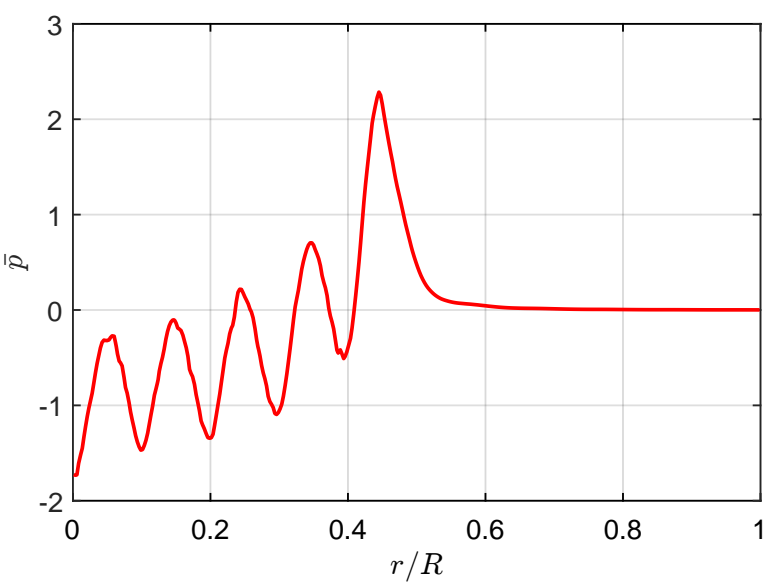

(c) contact force: config. $B$

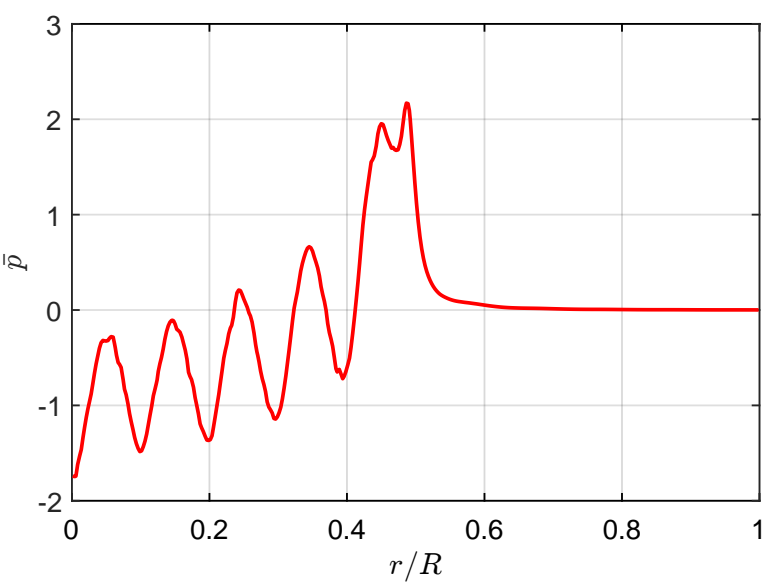

(e) contact force: config. $C$

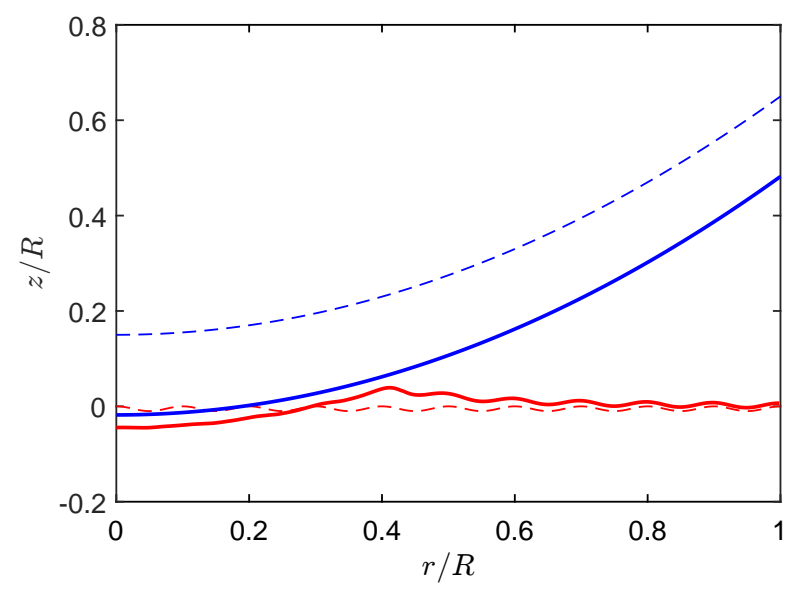

(b) surface profile: config. $A$

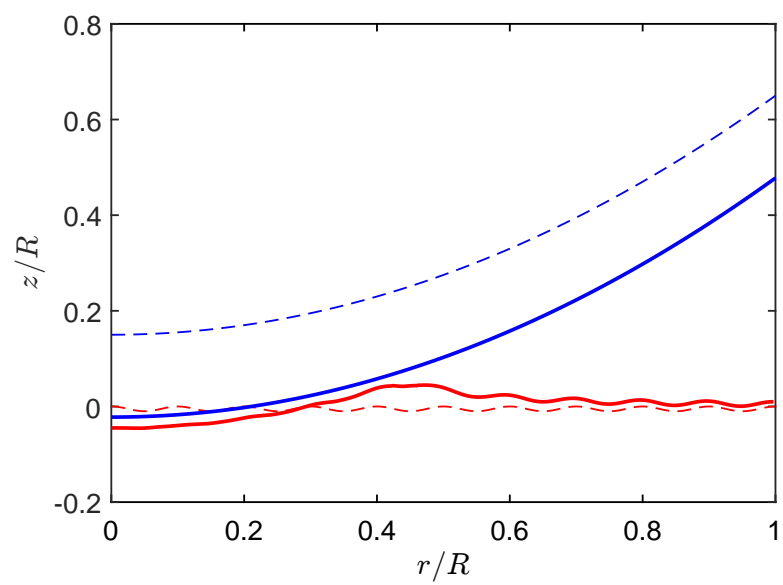

(d) surface profile: config. $B$

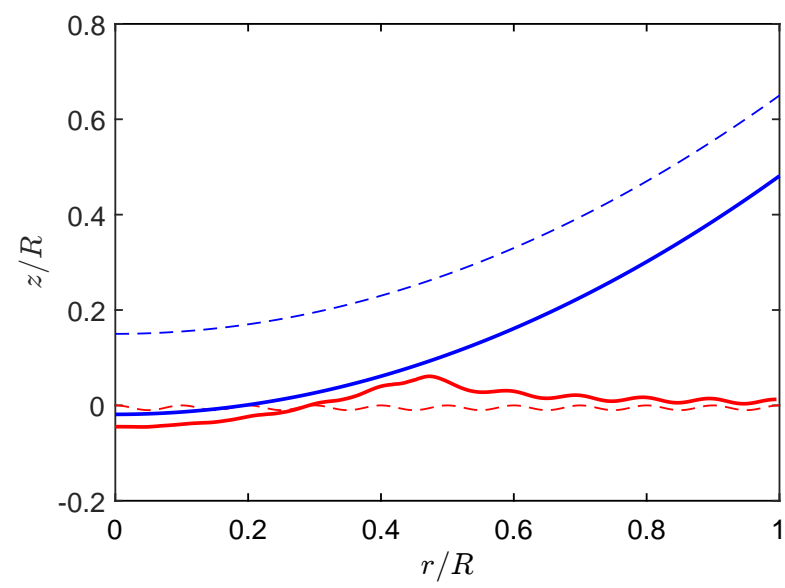

(f) surface profile: config. $C$

Fig. 18: Contact forces and corresponding surface profiles for three different configurations indicated in fig. $21 \mathrm{~b}$. 


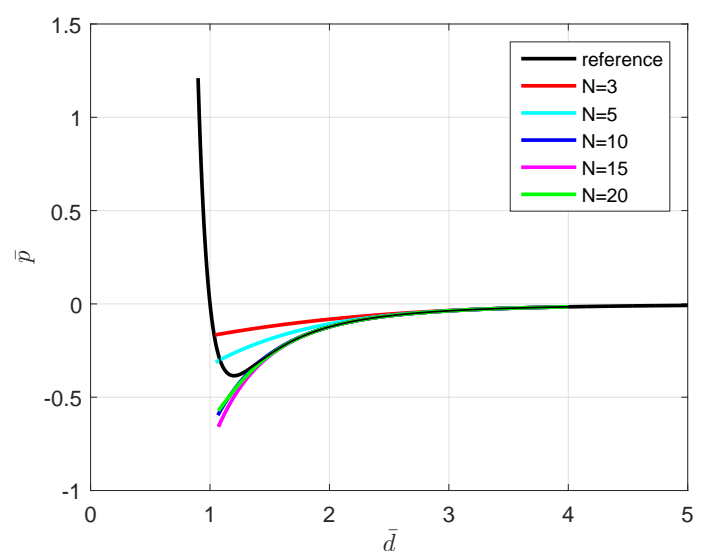

(a) $\quad \bar{d}_{0}=4$, Method- 1

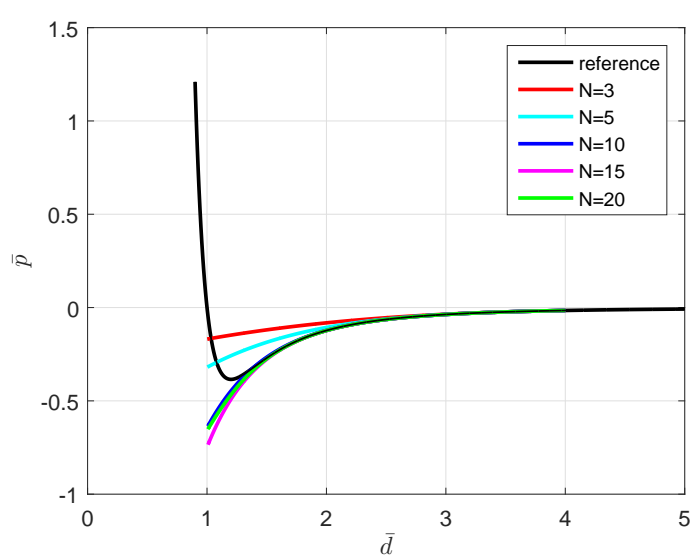

(c) $\bar{d}_{0}=4$, Method-2

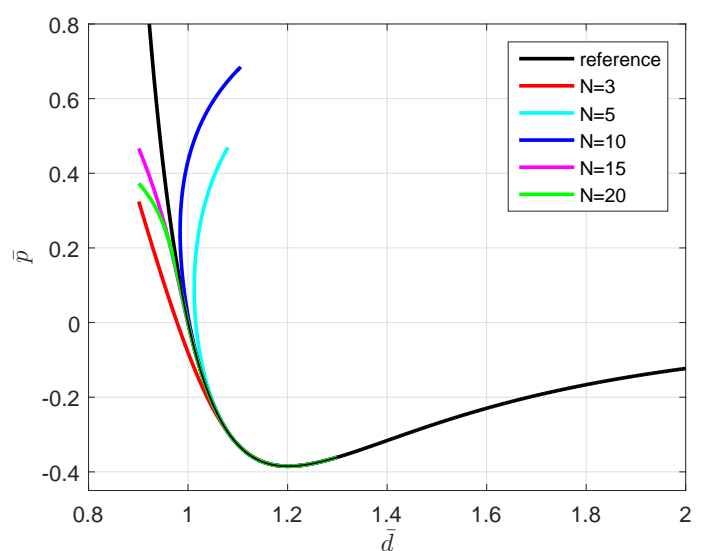

(b) $\bar{d}_{0}=1.3$, Method- 1

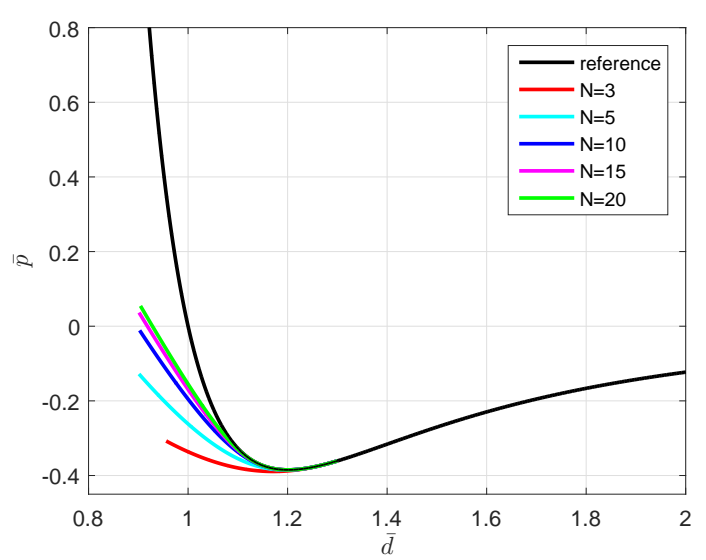

(d) $\bar{d}_{0}=1.3$, Method-2

Fig. 20: A single ANM branch to trace the normalized LJ interaction eq. (51)

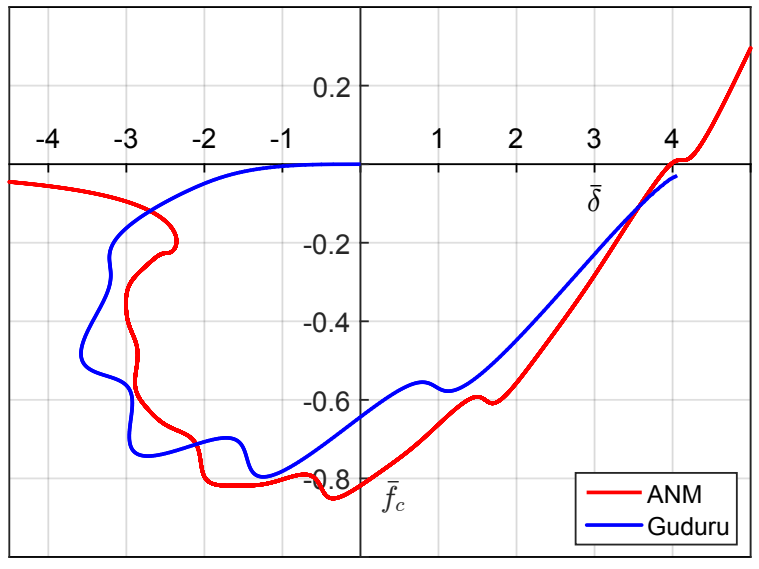

(a)

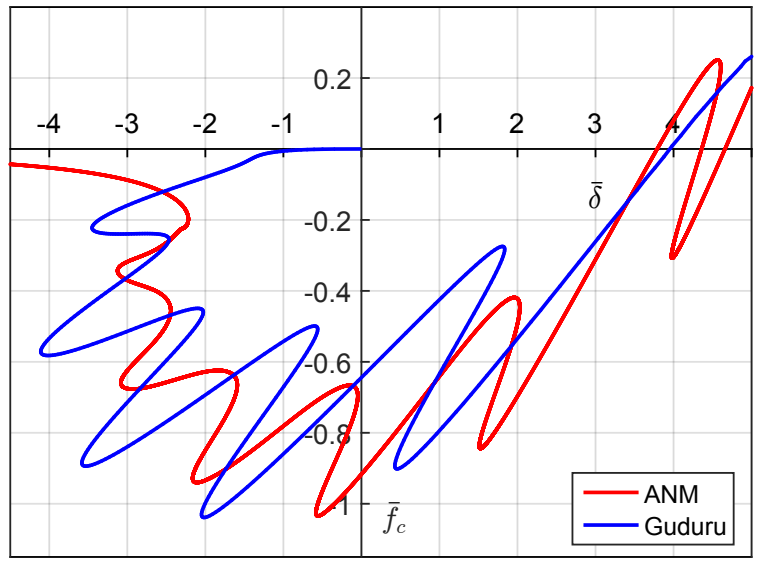

(b)

Fig. 21: Comparison of the ANM results with the Guduru theory: (a) $B / R=0.1, A / B=0.01$, (b) $B / R=0.1, A / B=0.05$ 